\title{
WestVirginiaUniversity
}

THE RESEARCH REPOSITORY @ WVU

Graduate Theses, Dissertations, and Problem Reports

2000

\section{Limits of behavioral control by temporally extended response -reinforcer relations}

Todd Matthew Myers

West Virginia University

Follow this and additional works at: https://researchrepository.wvu.edu/etd

\section{Recommended Citation}

Myers, Todd Matthew, "Limits of behavioral control by temporally extended response -reinforcer relations" (2000). Graduate Theses, Dissertations, and Problem Reports. 3181.

https://researchrepository.wvu.edu/etd/3181

This Dissertation is protected by copyright and/or related rights. It has been brought to you by the The Research Repository @ WVU with permission from the rights-holder(s). You are free to use this Dissertation in any way that is permitted by the copyright and related rights legislation that applies to your use. For other uses you must obtain permission from the rights-holder(s) directly, unless additional rights are indicated by a Creative Commons license in the record and/ or on the work itself. This Dissertation has been accepted for inclusion in WVU Graduate Theses, Dissertations, and Problem Reports collection by an authorized administrator of The Research Repository @ WVU.

For more information, please contact researchrepository@mail.wvu.edu. 
Limits of Behavioral Control by Temporally Extended Response-Reinforcer Relations

Todd M. Myers

\author{
A Dissertation \\ submitted to \\ the Eberly College of Arts and Sciences \\ in partial fulfillment of the requirements \\ for the degree of \\ Doctor of Philosophy \\ in \\ Psychology \\ Michael Perone, Ph.D. (Chair) \\ Kennon A. Lattal, Ph.D. \\ David W. Schaal, Ph.D. \\ Georg Eifert, Ph.D. \\ Richard T. Walls, Ph.D. \\ Department of Psychology \\ Morgantown, West Virginia \\ 2000
}

Keywords: molar and molecular response-reinforcer relations, correlation, contiguity, delay, lever press, rats

Copyright 2000 Todd M. Myers 


\begin{abstract}
Limits of Behavioral Control by Temporally Extended Response-Reinforcer Relations
\end{abstract}

Todd M. Myers

Three experiments were performed to determine the extent to which the behavior of rats can be controlled by response-reinforcer relations that are extended in time. In Experiment 1, bonus pellets delivered at the end of the session were contingent upon a shift in choice responding within the session. Experiment 2 examined control of aggregated responses by a delayed consequence over a much shorter time period than an entire session. The reinforcing efficacy of bonus pellets was assessed using a chained-schedule procedure. The relation between aggregated responses and a delayed reinforcing consequence was assessed several times per session and with shorter delays than in Experiment 1. Experiment 3 used an adjusting-delay procedure to assess whether differential reinforcer magnitudes have a differential effect on choice behavior when the delay between choice and subsequent reinforcement is equal for the two alternatives. The experiment was designed to determine the longest delay at which differential reinforcement is effective. Taken together, these three experiments were designed to determine the extent to which aggregated responses may be controlled by aggregated reinforcers or a single reinforcing event, and the extent to which a single response may be reinforced by its delayed consequence. Experiment 1 failed to produce reliable control of choice responding by the post-session consequence. Experiment 2 established control of responding by the delayed reinforcer, but such control was reliable for all rats only at delays of $40 \mathrm{~s}$ and less. Experiment 3 was unsuccessful in establishing discriminated choice performance by the large reinforcer, even at short delays, preventing the determination of the temporal limit of control by differential reinforcer magnitude. Overall, the results of this series of experiments suggest that the operant behavior of rats can be controlled by delayed consequences, but a finite limit to such control exists. It seems that reinforcers delayed on the order of several minutes or more are unlikely to control the behavior that produces them. Thus, response-reinforcer contiguity determines whether response-reinforcer correlations can control behavior. 


\section{Dedication}

To science and understanding, skepticism and wonder, curiosity and discovery. 


\section{Acknowledgments}

First and foremost, I thank my wife, Teri, for practically everything.

My family has provided continual encouragement and support without which I would not have begun or completed this work. Thank you.

I sincerely thank the members of the examining committee (Georg Eifert, Andy Lattal, Mike Perone, Dave Schaal, and Dick Walls) for helpful and insightful comments and suggestions.

Special thanks to Mike Perone for his constructive editorial remarks on previous drafts of this manuscript and for his guidance throughout my tenure at West Virginia University.

Thanks to Kristen Howell for assisting in data collection. 


\section{Table of Contents}

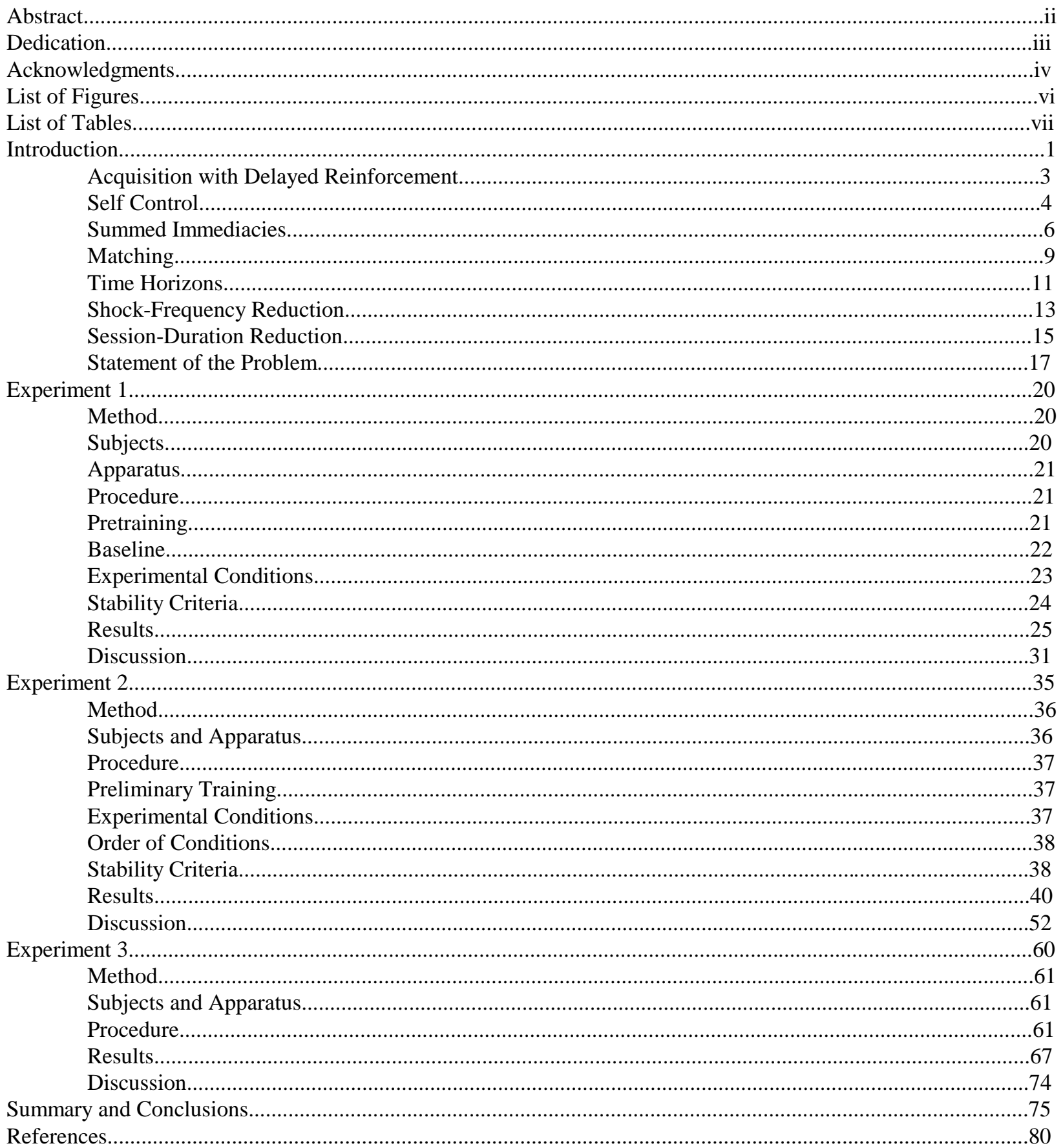




\section{List of Figures}

Figure 1. Experiment 1. Percentage shift in responding in each condition...................................29

Figure 2. Experiment 1. Number of bonus pellets for each session...............................................33

Figure 3. Experiment 2. Median baseline, initial-link, and terminal-link response rates...............42

Figure 4. Experiment 2. Correlation of baseline and initial-link responses per minute.................44

Figure 5. Experiment 2. Median initial-link minus baseline responses per minute........................47

Figure 6. Experiment 2. Median initial-link minus baseline latency, in seconds...........................51

Figure 7. Experiment 3. Proportion of large, left, and active choices..........................................68 


\section{List of Tables}

Table 1. Experiment 1. The order of conditions, response rates on the left and right levers, percentage of left lever responses, the average number of bonus pellets, and the number of sessions in each condition.

Table 2. Experiment 2. Order of conditions, the number of sessions in each, and the median and interquartile ranges of baseline, initial-link, and terminal-link response rates for each condition. Also shown are the median and interquartile range of difference scores (initiallink minus baseline responses per min) in each condition

Table 3. Experiment 2. Order of conditions and the median and interquartile ranges of baseline and initial-link latency for each condition, in s. Also shown are the median and interquartile range of difference scores (initial-link minus baseline latency), in s....

Table 4. Experiment 3. The order of conditions and number of sessions for each rat.

Table 5. Experiment 3. Individual and summed probabilities of left and right choices

following a particular position-magnitude combination. .72 
Introduction

Operant behavior is behavior controlled by its consequences. The maintaining consequence is called a reinforcer and the process by which the behavior is strengthened or maintained is called reinforcement. Control of operant behavior by molecular, or short-term, relations between response and reinforcer is not in dispute. However, the extent to which behavior may be controlled by more molar, or long-term, response-reinforcer relations is not clear. Molar consequences are those that are temporally delayed, remote, or diffuse. Molar behavior may be said to involve an aggregation of responses over time, rather than any one response. In the most extreme case, molar relations involve aggregated responses and aggregated consequences. In such cases, the assumption is that the correlation between responses and reinforcers over time is sufficient to control behavior. Molar relations between responding and its consequences have received considerable attention in the conceptual arena (Baum, 1973;

Hineline, 1977; Herrnstein, 1970), but empirically, control by molar relations has been examined only on a limited scale and by relatively few experiments.

The theoretical distinction between molecular and molar response-reinforcer relations is sometimes ambiguous, and given that short-term or long-term relations between response and reinforcer may control behavior either separately or in combination, contention arises over the appropriate scale of analysis (Hineline, 1984). The molecular view emphasizes responsereinforcer relations that occur at relatively discrete moments. On the other hand, the molar view emphasizes overall correlations between aggregates of responses and aggregates of reinforcers. Thus, by "transcending the momentary" (Baum, 1973, p.147) the molar view requires some assumptions regarding the ability of organisms to respond to aggregated events. In other words, 
the molar view assumes "an averaging or integrating capability on the part of the organism" (Baum, 1973, p.148). The exact time scale over which such integration may occur is unclear.

The extent to which behavior can be controlled by molar response-reinforcer relations is a question of empirical, conceptual, and applied significance. Determining the limits of control by molar relations would allow researchers to design procedures more likely to establish control over behavior. Once those limits are determined, relevant conceptual issues could be clarified. For example, many models of foraging behavior in natural settings assume that behavior is sensitive to events extended over a 24-hr period (Belovsky, 1978; Kamil, 1978). Further, finding the limits of control by molar relations in a particular species under laboratory conditions suggests that such limits may exist for many species under a variety of conditions. From the applied end, obvious practical benefits could be achieved with a fuller understanding of the efficacy of molar relations in controlling operant behavior. Indeed, many response-reinforcer relations in the natural world are molar (e.g., a comfortable retirement results from the cumulative effect of numerous spend-or-save decisions over decades). By establishing which relations are effective in changing behavior and which are not, a more prudent use of resources and more significant behavioral improvements in applied settings could result.

Experimental efforts to analyze explicitly molar and molecular control of behavior have not been able to settle the issue. This is because the issue is complex and cuts across many behavioral phenomena that appear to demonstrate the primacy of the molar or molecular view. However, most of these research areas have yielded findings that can be described within a molar or molecular explanatory framework and, therefore, do not strictly support one or the other view (for example, one-factor and two-factor explanations of avoidance behavior; Sidman, 1962; 
Herrnstein, 1969; Herrnstein \& Hineline, 1966; Hineline, 1981; Dinsmoor, 1977; Anger, 1963; Myers \& Perone, 1998). Below I shall discuss several research areas that bear on the present questions of molar control. Acquisition with Delayed Reinforcement

One assumption of the molar view is that a correlation between response and reinforcer is sufficient to control behavior, even when the response is temporally separated from the reinforcer it produces. Studies showing the acquisition of operant behavior under conditions of delayed reinforcement can be viewed as supporting this aspect of molar control. However, these studies demonstrate that reliable acquisition occurs only at short delays (approximately $30 \mathrm{~s}$ and less) thereby supporting the importance of response-reinforcer contiguity, or immediacy, in controlling operant behavior. In other words, a correlation between responding and reinforcement is sufficient to control behavior, assuming that the time period over which these correlations occur is short.

Critchfield and Lattal (1993) demonstrated acquisition of responding in rats using reinforcers delayed by $30 \mathrm{~s}$. The target response was defined as breaking a photobeam near the ceiling of the chamber. When such a response occurred, a food pellet was scheduled to be delivered $30 \mathrm{~s}$ later. This procedure ensured that responses were followed by food only after $30 \mathrm{~s}$ without a response. All six rats acquired the photobeam response and it was maintained at approximately 2 responses per min. The chamber also contained a response lever, but pressing it had no scheduled consequence. Responding on the lever did not increase during the acquisition of the photobeam response, showing that the result was not due to a general increase in activity resulting from the presentation of food. Using similar procedures and delays up to $30 \mathrm{~s}$, Lattal 
and Gleeson (1990) demonstrated acquisition of key-pecking in pigeons and acquisition of leverpressing in rats. Lesage, Byrne, and Poling (1996) provided support for acquisition of lever pressing under delayed water reinforcement using resetting delays as high as 16 s. Wilkenfield, Nickel, Blakely, and Poling (1992) used slightly different delayed reinforcement procedures and obtained similar findings. Lattal and Metzger (1994) extended the generality of acquisition under delayed reinforcement using fish and visual reinforcement. Although acquisition of behavior under delayed reinforcement appears to be a robust phenomenon, reliable acquisition with reinforcers delayed more than $30 \mathrm{~s}$ from the target response has not been demonstrated. Using resetting delays of 60 s, Snycerski, Laraway, Byrne, and Poling (1999) failed to produce acquisition of lever pressing. Molar theories are based on the integration of events over a much longer time period, for example, sessions of $30 \mathrm{~min}$ or more. A period of 30 or $60 \mathrm{~s}$, then, seems too short to support the degree of molar control implicit in such theories.

\section{$\underline{\text { Self Control }}$}

Studies of self control have demonstrated the importance of response-reinforcer delay in determining reinforcer efficacy. These studies have also shown that larger magnitude reinforcers are more effective. Thus, immediate reinforcers control choice behavior more effectively than delayed reinforcers and, at equal delays to reinforcement, larger reinforcers are more effective.

Studies of self control examine choice by placing reinforcer magnitude and reinforcer delay in opposition (for a review see Logue, 1988). The choice presented is between a smaller, more immediate reinforcer and a larger, more delayed reinforcer. Preference for the large reinforcer is referred to as self control and preference for the small reinforcer is referred to as impulsivity. These two descriptive labels, self control and impulsivity, may be related to the 
principle of optimization (Schoemaker, 1982; Rosen, 1967).

Optimization theory predicts that animals will maximize some reinforcer (or resource) over an extended period of time. For example, the pattern of choice that produces the greatest amount of food over a 24-hr period or the greatest number of reinforcers per minute (or per response) is said to be maximally efficient; it is optimal with respect to that aggregate measure of reinforcement. Of course, because moment-to-moment response-reinforcer relations are not captured by the aggregate measures, optimization implies that the temporal relations between individual responses and reinforcement are relatively unimportant in controlling behavior.

In the self-control paradigm, choosing the larger reinforcer results in a greater rate of reinforcement when the rate of trial presentation is controlled. This pattern of responding is observed often when the magnitude:delay ratio of the larger reinforcer is greater than that of the smaller reinforcer. For example, presented with a choice between six pellets presented after a 3-s delay (a 2:1 ratio) and 1 pellet presented after a 1-s delay (a 1:1 ratio), rats would likely prefer the larger, delayed reinforcer. If the ratio shifted in favor of the smaller reinforcer, choice would be expected to shift in favor of the smaller reinforcer (e.g., 6 pellets delayed $12 \mathrm{~s}$ versus 1 pellet delayed $1 \mathrm{~s}$ ). This predictive model of individual choices based on the magnitude:delay ratio of the alternatives takes account of both of these potent reinforcement variables. However, this model may not predict choice accurately for all species (van Haaren, van Hest, \& van de Poll, 1988) or when one alternative is greatly delayed (Ito \& Oyama, 1996), but the basic tenets of the model are not in dispute. Overall, the results show that choice is determined by the interaction of response-reinforcer delay and reinforcer magnitude. Because these studies demonstrate that reinforcer efficacy decays rapidly with delay, the data imply, but do not identify, a maximal delay 
at which a single reinforcing event can control a choice response. The data do not suggest that choice is controlled by several reinforcers extended over time.

$\underline{\text { Summed Immediacies }}$

Much empirical support for the control of choice by aggregate reinforcers comes from studies applying the summed-immediacies model. Also referred to as the sum-of-reciprocals model, it predicts a particular choice by the summed immediacies (the reciprocals of the delays) to the next few reinforcers, timed from the moment of choice. I will use an example to illustrate application of the model. Shull, Spear and Bryson (1981) arranged a choice between a schedule providing food at variable intervals and a second schedule that provided food at different fixed intervals. Two keys were present, a food key and a switching key. When the food key was green, the variable-interval schedule operated and arranged reinforcement at intervals averaging $113 \mathrm{~s}$. A peck to the white switching key turned off the switching key and changed the color of the food key to red. The red component schedule was in effect for 240 s. Pecking the red food key produced food at different intervals, but the pattern of food presentations was constant in each condition. In one condition, programmed delays to reinforcement in red (timed from the peck to the switching key) were 30,195 , and $210 \mathrm{~s}$. The sum of the reciprocal delays to reinforcement was $1 / 30+1 / 195+1 / 210=0.033+0.005+0.005=0.043$. In another condition, programmed delays were 30,45 , and $210 \mathrm{~s}$, and the sum of reciprocals was $1 / 30+1 / 45+1 / 210=0.033+$ $0.022+0.005=0.060$. The only difference between these two conditions was in the delay to the second reinforcer in the red component. For all three pigeons, the rate of switching into the red component was higher in the latter condition, presumably due to the shorter delay to the second reinforcer. Rates of switching were similar, however, in a condition providing 30, 60, 195, and 
$210 \mathrm{~s}$ delays as they were in a condition arranging delays of $30,60,75$, and $210 \mathrm{~s}$. In this latter comparison, the differential delay to the third reinforcer did not differentially affect choice of the red component. Nevertheless, the data were consistent with a model of choice based on the delay to more than one reinforcer timed from a particular choice point. Thus, aggregate reinforcers may control choice. The model is particularly appealing because it acknowledges the importance of response-reinforcer delay in the control of choice by aggregate reinforcement.

The results of other choice procedures have supported the summed-immediacies approach. Several investigators have arranged simultaneous choice between escalating and fixed schedules. The escalating schedule requires increasing amounts of time (or effort) to complete. The schedule requirement begins at a low value and increases each time a reinforcer is earned. The requirement of the fixed schedule is constant and is higher than the minimum requirement of the escalating schedule. Molecular and molar predictions are the same under these circumstances. The subject should choose the escalating schedule several times until the requirement of the escalating schedule approximates that of the fixed schedule, then choose the fixed schedule for the remainder of the session. This pattern minimizes the delay to the next reinforcer and maximizes the reinforcement rate, favoring neither a molar or molecular view. However, in the presence of a reset contingency on the fixed schedule, molar and molecular predictions differ, allowing a determination of whether one or more reinforcers is likely controlling choice.

Under reset conditions, completing the requirement of the fixed schedule produces a reinforcer and resets the requirement of the escalating schedule to its minimum. If choice were controlled simply by the delay to the next reinforcer, the subject would choose the escalating schedule several times until the requirement of the escalating schedule approximated that of the 
fixed schedule, then choose the fixed schedule, thereby resetting the escalating schedule. The pattern would then be repeated. This pattern of choice minimizes the delay to the next reinforcer at every choice opportunity. In contrast, if choice were controlled by the next few reinforcers, the subject would reset the escalating schedule sooner and keep the requirement close to its minimum. This would entail choosing the fixed schedule when the requirement is lower on the escalating schedule, opting for a less immediate reinforcer in the short term but increasing reinforcement rate over the next few reinforcers and, thus, over the session.

This procedure can assess whether an account based on summed immediacies to reinforcement or optimization of reinforcement (in terms of reinforcers per response or unit time) provides a better description of the data because each predicts a different switch point. In many cases, the data have supported the summed immediacies model using about four reinforcers in the calculations (Neuman, Ahearn, \& Hineline, 2000; Wanchisen, Tatham, \& Hineline, 1988; Mazur \& Vaughan, 1987; Hineline \& Sodetz, 1987). However, some studies using similar procedures with humans and primates support an optimization account (Jacobs \& Hackenberg, 1996; Hackenberg \& Axtell, 1993; Hodos \& Trumbule, 1967). Both views support the role of aggregate reinforcers in controlling choice but neither provides an index of the time period over which such control occurs. The results of Mazur and Vaughan (1987) suggest that this period may be rather short. Using similar choice procedures between fixed and escalating schedules, Mazur and Vaughan interpolated an intertrial interval between each reinforcer and the next choice period. Choices tended to minimize the delay to the next one or two reinforcers when the intertrial interval was long (50 s) and tended to minimize the delay to the next four reinforcers with short intertrial intervals ( 25 and $0 \mathrm{~s}$ ). Optimization did not account for these results, 
predicting no change with changes in intertrial interval. The results suggest that the number of aggregate reinforcers controlling choice in these procedures may depend on the time period over which those reinforcers are presented. Overall, these data support the view that the temporal separation between a response and a reinforcer determines the efficacy of that reinforcer, despite its serial position within a sequence of reinforcers.

\section{$\underline{\text { Matching }}$}

In the extreme case, molar relations are those that involve aggregate responses and aggregate reinforcers over long time periods. Long-term correlations between these aggregate measures, response rate and reinforcement rate, have been addressed directly by studies of matching. The procedures typically arrange a simultaneous choice between two independent schedules of reinforcement that provide reinforcers at variable intervals. Manipulating the reinforcement rate of one alternative changes the relative reinforcement rate of that alternative. One reliable finding is that the proportion of responding allocated to an alternative (measured in terms of number of responses or time spent responding) is a direct function of the proportion of reinforcement earned by responding on that alternative (Herrnstein, 1970; Logue \& de Villiers, 1978; de Villiers, 1974; for a comprehensive review see Herrnstein, 1997). One interpretation of these findings is that aggregated choice responding on each alternative is controlled by the aggregated reinforcement rate provided by each alternative over the entire session. Thus, implicit in this interpretation is that choice behavior is sensitive to aggregate response-reinforcer relations extended over long periods of time (sessions of $30 \mathrm{~min}$ or more). Certainly the choice data are orderly at this molar level, but the results do not illuminate the mechanism that produces this overall relation between relative responding and relative rate of reinforcement. Indeed, the 
matching law does not predict a particular choice at a particular moment. The matching law predicts that each of the choices, overall, will comprise a proportion of all choices in direct relation to the proportion of reinforcers it provides.

Melioration takes account of local changes in reinforcer probability for each alternative, and may provide the mechanism for choice responding and the moment-to-moment predictions of choice that matching does not. Melioration accounts for local changes in choice by examining local changes in reinforcer probability. As the reinforcement rate for an alternative increases, the proportion of responding allocated to it increases. However, as time spent responding to one alternative increases, the probability of reinforcement increases on the other alternative, eventually producing a shift in choice to that alternative. In this way, behavior is believed to track relatively local shifts in reinforcement rate, thereby producing the commonly observed matching relation, an overall correlation between relative response rate and relative reinforcement rate.

The principle of melioration states that choice is determined by "a comparison of the average returns from the alternatives" (Herrnstein, 1997, p. 240). Thus, melioration assumes integration of events over time by asserting that choice is controlled by recent responding on each alternative and recent reinforcement received from each. However, the principle says little with respect to the precise time period over which such integration occurs. The time frame may be large or small and neither matching nor melioration addresses the issue directly.

Indirect support for a limited period of integration comes from the results of some melioration and matching studies that placed optimization and melioration in opposition. Although most studies of matching have not been designed to separate control by melioration from optimization, those that have attempted to do so have shown that melioration appears to 
control choice (Herrnstein \& Heyman, 1979; Herrnstein \& Vaughan, 1980; Mazur, 1981; Vaughan, 1981). Relative responding tends to stabilize at a point where the probability of reinforcement is approximately equal for the two alternatives at any time, despite producing a decrease in overall reinforcement rate. Thus, the pattern of choice produced is sub-optimal at the level of the session (similar to the results from the aforementioned experiments examining self control and the summed-immediacies model). This implies that the time frame over which integration occurs is shorter than the length of the session. At present, the data from the matching literature seem to support control of choice by local changes in relative probability of reinforcement over a relatively short time period, rather than supporting a view of choice allocation controlled by overall response-reinforcer correlations.

\section{$\underline{\text { Time Horizons }}$}

Some studies have shown that delayed events can affect not just a single response, but an aggregation of responses across time, and therefore bolster the claim that molar control of aggregated responses is possible. Bacotti (1976) demonstrated control of within-session responding by manipulating the delay between the end of the session and a subsequent feeding (but cf. McSweeney, Hatfield, \& Allen, 1991). Rats produced food pellets on a variable-interval (VI) 60-s schedule of reinforcement. A response (key or lever press) was reinforced after an average of $60 \mathrm{~s}$ elapsed. Each session lasted $1 \mathrm{hr}$. In the immediate-feeding condition, the postsession feeding (i.e., the daily food ration) was given within 2 min of the end of each session. In the delayed-feeding condition, the feeding was presented $1 \mathrm{hr}$ after each session. Five rats were exposed to both conditions once. Six other rats were exposed to the immediate-feeding condition twice, each separated by exposure to the delayed-feeding condition. Conditions were in effect for 
a minimum of 15 sessions. For every rat, response rates during the session were higher under the delayed-feeding condition. Although a general suppression of responding throughout the sessions was obvious in the immediate-feeding condition, the decrease was most apparent near the end of the sessions. The percent increase in response rate produced by changing to the delayed-feeding condition ranged from about $20 \%$ to $150 \%$. These results demonstrate that the delay between the end of the session and the feeding affected within-session operant responding. When feedings were delayed about 2 min from the end of the session, response rates were lower than when feedings were delayed about $60 \mathrm{~min}$. Thus, an event at the end of a 60-min session affected behavior throughout the session. This implies that aggregated responses can be controlled by a greatly delayed event.

Timberlake, Gawley, and Lucas (1988) also demonstrated that a remote event can affect aggregated responses. These experimenters manipulated the delay to a less costly food source and measured responding to a more costly food source. The chamber had two levers, each on a separate side. The levers were separated by a partition and weight-sensing floor panels measured the location of the rat. A progressive-ratio (PR) schedule was available on one lever. The PR schedule began at a response requirement of one and the requirement increased by one response after each pellet delivery. Access to a fixed-ratio (FR) 1 schedule on the other side of the chamber was contingent on leaving the side of the chamber with the PR schedule or not responding on the PR schedule for $15 \mathrm{~s}$, or entering the side of the chamber with the FR schedule, whichever occurred first. After spending a cumulative time of 2, 4, 8, 16, 32, or 64 min away from the PR schedule, the FR 1 schedule became operative. The FR 1 schedule provided a pellet for each response and was operative for $6 \mathrm{~min}$. The session ended at the end of the 6-min 
period. Thus, rats were presented with a choice between a schedule available now that required increasing amounts of effort and a schedule that required little effort but was not available until after a delay. The goal was to determine whether rats would cease responding on the PR schedule sooner when the delay to the FR 1 schedule was short. Results showed a change in PR schedule performance as a function of the delay to the onset of the FR 1 schedule. When the FR 1 schedule was delayed 64 min, performance on the PR schedule was unaffected. At a delay of 32 min, two of the four rats earned fewer reinforcers on the PR before initiating the delay to the FR. For another rat, only delays equal to or less than 16 min affected PR performance. The PR performance of the fourth rat was affected only at delays equal to or less than $8 \mathrm{~min}$. Despite the individual differences, the overall results appeared to demonstrate control of aggregated responses by an event delayed 8 or more min from those responses. However, because entering the FR side of the chamber could be reinforced immediately, the procedure did not ensure a temporal separation between responding and reinforcement, complicating a strictly molar interpretation. Moreover, control-group data (from rats receiving no access to the FR 1 schedule) suggest that changes in PR performance may have resulted from changes in session duration alone.

Shock-Frequency Reduction

Rather than indirectly assessing molar control, a few studies have attempted to explicitly establish molar control of behavior by arranging experiments that minimize molecular responsereinforcer relations while providing molar relations designed to control responding. However, these studies purporting to show molar control do not conclusively do so because a molecular account of performance remains plausible, even when steps to eliminate such interpretations are 
undertaken. Herrnstein and Hineline's (1966) study of shock-frequency reduction is a case in point.

Herrnstein and Hineline (1966) designed a procedure that allowed responses to reduce the frequency of shock in the absence of fixed delays between responses and shocks. Responding reduced overall shock frequency but a response could be followed by a shock immediately or after a brief period of time. The procedure employed two independent shock schedules. Both schedules arranged shock at variable intervals but only one schedule was in control at any given time. In most cases, the frequency of shock programmed by each schedule differed. In the absence of responding, shocks were delivered by the higher-frequency schedule. A single response switched control from the higher-frequency schedule to the lower-frequency schedule. Additional responses during the lower-frequency schedule were ineffective, producing only the feedback stimulus (an audible relay click). The lower-frequency schedule remained in control until the next scheduled shock was delivered, at which time the lower-frequency schedule was suspended and the higher-frequency schedule was reinstated. The difference in shock frequency between the two schedules determined the maximum obtainable shock-frequency reduction.

Herrnstein and Hineline (1966) programmed reductions of 0, 3, 6, and 12 shocks per min. In the 0 condition the frequency of shock was the same under the two schedules, so responses could not reduce the frequency of shock. Responding extinguished under this condition. In the other conditions, in which responding did reduce shock frequency, responding was maintained and the rate of responding was directly related to the degree of shock-frequency reduction. Herrnstein and Hineline's results suggest that the molar consequence, overall shock-frequency reduction, reinforced responding. In other words, aggregated responses were controlled by 
aggregated consequences. Despite attempts to minimize immediate consequences of responding, Herrnstein and Hineline's procedure allowed for molecular interpretations of behavioral control. Most, but not all, responses led to immediate increases in the delay to the next shock. Thus, overall shock-frequency reductions were achieved by cumulative short-term increases in the delay to shock that resulted from responding. Furthermore, because shock rates were so high, the experiment provided little support for the molar view. Specifically, in the condition that programmed the smallest reduction ( 3 shocks per min), responding changed the average rate of shock from 1 every $10 \mathrm{~s}$ to 1 every $20 \mathrm{~s}$. Thus, the experiment arranged shifts in shock rate over very short time periods and neither required nor demonstrated control by greatly delayed events.

\section{$\underline{\text { Session-Duration Reduction }}$}

Mellitz, Hineline, Whitehouse, and Laurence (1983) examined whether the avoidance behavior of rats could be controlled by reductions in the duration of avoidance sessions. A single avoidance schedule was in effect and a response on either of two levers was effective in postponing the next shock. However, each press on one of the levers, the conjoint lever, postponed shock and subtracted $1 \mathrm{~min}$ from the scheduled session duration. Each session was programmed to last $152 \mathrm{~min}$. The goal was to see whether the rats would prefer the conjoint lever, thereby demonstrating sensitivity to the molar consequence of session-duration reduction. Across blocks of sessions, the position of the conjoint lever (left or right) was reversed to see if response preference would track the conjoint lever. To reduce the likelihood that a response on the conjoint lever would be followed closely in time by the termination of the session, the session-reduction contingency was disabled during the final $2 \mathrm{~min}$ of each session. Of the five rats exposed to the procedure, only two showed any evidence of control by the conjoint 
contingency. For one of these rats, choice behavior tracked the conjoint lever across one reversal and then persevered on one of the levers for the remainder of the experiment. The other rat that showed evidence of control by the session-reduction contingency tracked the conjoint lever across four conditions. Although the procedure did not reliably produce control by the molar contingency of session-duration reduction for even a majority of rats, the results provide support for the possibility of behavioral control by extremely molar response-reinforcer relations.

There appear to be two assertions implicit in the study by Mellitz et al. (1983). One is that aggregated responses can be controlled by aggregated reinforcers. That is, the degree to which the avoidance session was reduced in duration was a direct function of the responses cumulated on the conjoint lever. The second assertion is that the reinforcer can be temporally separated from the effective responses and still control behavior. After all, responses to the conjoint lever for 2 min prior to the end of the session were ineffective in reducing the duration of the session. These two assertions, sensitivity to aggregated response-reinforcer relations and control by a delayed consequence, can be tested either separately or in combination. Doing so would provide a direct test of the relations necessary for the kind of molar control that has been proposed.

Due primarily to two procedural weaknesses, Mellitz et al. (1983) did not conclusively demonstrate molar control of choice. First, although the session-reduction contingency was disabled during the final 2 min of each session, responses on the conjoint lever could have been followed closely in time by the termination of the session. In other words, the procedure did not ensure that the end of the session was temporally separated from conjoint lever responses. Thus, the delayed consequence of session termination may not have been delayed at all. Indeed, Mellitz et al. failed to examine responses just prior to the end of the session, allowing this more 
molecular interpretation to remain plausible. Secondly, the stability criteria used to assess preference for the conjoint lever across conditions were biased in favor of showing a preference for the conjoint lever. Conditions were in effect for a minimum of five sessions but were not ended until the rat preferred the conjoint lever for three consecutive sessions. Assuming a rat had no reliable preference for the conjoint lever and responded approximately randomly across the two levers from session to session, this stability criterion would select preference for the conjoint lever as the representative terminal data for each condition. Furthermore, once an apparent preference for the conjoint lever was established, too few sessions were conducted to see if that preference was reliable or merely transient. This latter criticism is particularly troubling in light of the results of Verhave (1961). He arranged a similar avoidance procedure but with no sessionduration reduction. A response on either of two levers was equally effective in postponing shock. Verhave found that the preference, or bias, for one lever was often great and this preference shifted unpredictably from lever to lever within and between sessions. Some rats had strong biases for one lever by the end of the experiment, like some of the rats in the study by Mellitz et al. This underscores the importance of adopting rigorous stability criteria in experiments assessing choice.

\section{Statement of the Problem}

The extent to which behavior can be controlled by molar response-reinforcer relations is a question of conceptual and empirical significance. The question is fundamental to an understanding of how reinforcement exerts its response-strengthening effects and addresses directly the temporal limits of reinforcement operations. Current and long-standing debate has centered on the issue of whether correlations between responses and reinforcement, regardless of 
response-reinforcer contiguity, are sufficient to reinforce responding. Although molar and molecular views of behavioral control have been much debated, experimental efforts to analyze molar and molecular control explicitly have not been able to settle the issue. Studies purporting to show control by molar response-reinforcer relations have been met with skepticism by molecular theorists because a molecular account of performance remains, despite steps to eliminate such interpretations. In most molar accounts of behavior, there appear to be two assertions, both suggesting an ability on the part of the organism to integrate events occurring over an extended period of time. One is that aggregated responses can be controlled by aggregated reinforcers and the other is that a reinforcer can be temporally separated from the effective response and still control behavior. Both assertions can be tested either separately or in combination and doing so would provide a direct test of the kind of molar control that has been proposed (Baum, 1973; Mellitz et al., 1983; Herrnstein \& Hineline, 1966; Hineline \& Sodetz; 1987; Hodos \& Trumbule, 1967).

The present research was designed to improve upon previous research that attempted to demonstrate molar control of operant behavior. The research examined aggregate responsereinforcer relations as well as control by consequences temporally separated from the operant response, both assumed to be fundamental prerequisites to molar control.

In Experiment 1, bonus pellets delivered at the end of the session were contingent upon a shift in the distribution of responses maintained under two identical reinforcement schedules within the session. The experiment mimicked the one by Mellitz et al. (1983) but used positive as opposed to negative reinforcement. The goal was to examine whether a molar responsereinforcer relation could produce shifts in response preference. Aggregated responses produced 
aggregated reinforcers delivered at the end of the session.

Experiment 2 examined control of aggregated responses by a delayed consequence over a much shorter time period than an entire session. The reinforcing efficacy of bonus pellets was assessed using a chained-schedule procedure. Responses on a lever occasionally produced reinforcers. Periodically, a light came on. After a prescribed number of responses in the presence of the light, a delay to the delivery of bonus pellets was initiated. The response rate in the presence of the light should be higher than in the absence of the light when the delay to bonus pellets is short. At very long delays to the bonus, response rates during the light should approximate response rates in the absence of the light, indicating no control by the delayed consequence. This procedure allowed the relation between aggregated responses and a delayed reinforcing consequence to be assessed several times per session with shorter delays than in Experiment 1.

Experiment 3 used an adjusting-delay procedure to assess whether differential reinforcer magnitudes have a differential effect on choice behavior when the delay between choice and subsequent reinforcement is equal for the two alternatives. The experiment was designed to determine the longest delay at which differential reinforcement is effective. Rather than examining control by aggregate responses or reinforcers, Experiment 3 examined control of a single choice response by a single delayed consequence, providing a simpler test of control by delayed reinforcement than that provided by Experiments 1 and 2. Further, Experiment 3 was well suited to pinpoint more precisely the maximal delay of reinforcement capable of controlling behavior.

Taken together, these three experiments were designed to determine the extent to which 
aggregated responses may be controlled by aggregated reinforcers or a single reinforcing event, and the extent to which a single response may be reinforced by its delayed consequence. Thus, this series of experiments used various procedures to address whether the kind of molar control reported by Mellitz et al. (1983) is in fact possible.

\section{Experiment 1}

To examine sensitivity of aggregated responses to aggregated reinforcers, bonus pellets delivered at the end of the session were contingent on choice responding during the session. The procedure was modeled after that used by Mellitz et al. (1983) but used positive as opposed to negative reinforcement. Generally stated, this procedure arranged a molar consequence (accumulated bonus pellets) contingent on an aggregation of choice responses (degree of preference for a particular lever). Because sensitivity to the molar bonus contingency might be a function of the session duration, several rats were exposed to both 15- and 60-min sessions.

\section{Method}

\section{$\underline{\text { Subjects }}$}

Seven male Sprague-Dawley rats were used. The rats were approximately one year old at the start of the experiment. Each rat had a history of pressing the left lever under several simple schedules of reinforcement. The rats were maintained at approximately $80 \%$ of free-feeding weight through feedings given at least 20 min after being returned to their home cages.

\section{$\underline{\text { Apparatus }}$}

Seven operant chambers were used. The internal dimensions were $23.1 \mathrm{~cm}$ long by 20.3 $\mathrm{cm}$ wide by $18 \mathrm{~cm}$ high. Each chamber was housed in a sound-attenuating shell constructed of plywood; the front of the shell was open. The shell was equipped with a fan to supply ventilation 
and mask extraneous sounds. The side walls and ceiling of the operant chamber were constructed of Plexiglas and the front and rear walls of aluminum. The front wall had two levers, left and right. The levers were spaced $9 \mathrm{~cm}$ apart from center to center and protruded $2.8 \mathrm{~cm}$ into the chamber. A cue light was centered $2 \mathrm{~cm}$ above each lever. Centrally located at the top of the front wall was a houselight, $1.5 \mathrm{~cm}$ below the ceiling. The grid floor consisted of rods spaced $0.7 \mathrm{~cm}$ apart, aligned parallel to the front wall. The food aperture was $4 \mathrm{~cm}$ square. The bottom of the aperture was $0.8 \mathrm{~cm}$ above the grid floor. The left and right sides of the aperture were $2 \mathrm{~cm}$ from the left and right levers, respectively. A pellet dispenser was capable of delivering 45-mg Noyes pellets into a food tray accessible through the food aperture. In four of the chambers, pellet delivery was accompanied by an audible click. In the other three chambers a 0.1-s tone (Sonalert Model SC110) accompanied pellet delivery. Each chamber was situated in a separate cubicle in a large room. Control and recording operations were accomplished with microcomputers connected to the chambers by digital interfaces (Computer Boards, Inc., CIO-PDIS08), using a software system that supported timing to the nearest $0.01 \mathrm{~s}$.

\section{Procedure}

Pretraining. Each rat was exposed to a simple VI schedule of food reinforcement. The available lever was alternated across sessions until response rates were approximately equal on both levers when each was available separately. Because the rats had a history of reinforced responding on the left lever, additional sessions in which only the right lever was available were required for several rats. Initially, the VI value was $15 \mathrm{~s}$. By the end of pretraining the VI value was $60 \mathrm{~s}$. The first reinforcer after 60 min ended each session. 
Baseline. A concurrent VI 120-s VI 120-s schedule was arranged using a variation of Stubbs and Pliskoff's (1969) procedure to ensure that obtained rates of reinforcement from the left and right schedules were approximately equal within each session. Only one schedule was in operation at a time. At the start of the session and after each reinforcer delivery, the left schedule was selected with a probability of 0.5 . Each VI schedule consisted of 12 unique intervals averaging 60 s. The intervals were modeled after Fleshler and Hoffman's (1962) distribution. For each VI schedule, the 12 intervals were arranged in three, random orders to produce a list of 36 intervals. At the beginning of each session, a random starting point was selected for each VI schedule. Although the left or right schedule was equally likely to be in effect, the same schedule was not selected more than twice in succession. Thus, responding exclusively on one lever could produce, at most, two reinforcers before no more would be forthcoming. The first reinforcer delivery after 60 min ended the session. Because each session ended with a reinforcer and because of the interdependent nature of the schedules, the last reinforcer of the session was equally likely on the left and right levers.

A 5-s changeover delay (COD) ensured that rapidly switching between levers would not be reinforced. The COD was timed from the switch to the lever with a scheduled reinforcer (i.e., a press-press COD; cf. Shahan \& Lattal, 1998). The first press occurring at least $5 \mathrm{~s}$ after the switch produced the reinforcer only if no responses to the other lever intervened. A response to the other lever constituted a switch and reset the COD.

All rats were exposed to the concurrent VI 120-s VI 120-s schedule for at least nine 60min sessions. The rats were randomly assigned to either the 60-min group or the 15-min group. Rats in the 60-min group (Rats 3,4,9, and 10) were then placed directly on the experimental 
procedure. Rats in the 15-min group (Rats 1,2, and 8) were given a minimum of nine 15-min sessions before beginning the experimental conditions. These sessions were identical to the 60min sessions, except that the first reinforcer after 15 min ended the session. For each rat, the percentage of left lever responding across the last nine sessions was used as a baseline. The mean percentage of left responses of these nine sessions was used as the reinforcement criterion in the first experimental condition.

Experimental conditions. The concurrent schedule continued to operate as in baseline. In addition, bonus pellets were delivered at the end of the session if the percentage of left lever responding deviated from criterion in the prescribed direction: up, a shift toward the left, or down, a shift toward the right. For each full percentage point shift in the prescribed direction, two bonus pellets were delivered at the end of the session. If a bonus was earned, the first bonus pellet was delivered $1 \mathrm{~s}$ after the last reinforcer of the session and subsequent bonus pellets were delivered $1 \mathrm{~s}$ apart until all bonus pellets had been delivered. At that point the houselight was turned off. If no bonus was earned, the houselight was turned off after the final pellet delivery of the session. The bonus contingency was initially placed on the non-preferred lever (the right lever for all rats except Rat 1). Across conditions, the position of the bonus lever was alternated to determine whether responding would track the bonus contingency across repeated reversals. Specifically, responding should shift in favor of the left lever when bonus pellets are contingent on a shift toward the left and toward the right when the bonus is on the right.

The criterion for reinforcement changed with each change in condition. In the first experimental condition, the criterion was equal to the mean percentage of left lever responses in the baseline condition. Subsequent conditions set the criterion equal to the mean percentage of 
left responses in the immediately preceding condition. This allowed for frequent updating of the criterion throughout the experiment and increased the likelihood that a rat would contact the bonus contingency in each condition.

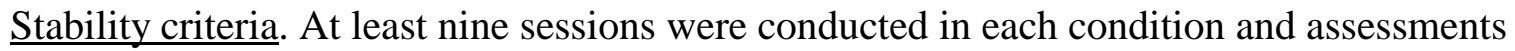
of stability were based on the last nine sessions. The last nine sessions were divided into three blocks of three sessions each. Performance was considered stable if the mean percentage of left responses for each of the three blocks showed no increasing or decreasing monotonic trend and the minimum and maximum block means differed by less than 10 . The mean percentage of left responses and the absolute response rates on the left and right levers had to appear visually stable. The bonus contingency had to be encountered at least twice in a condition (but not necessarily in the last nine sessions). In some cases, the bonus contingency was not encountered more than once despite several sessions (Rat 1, Condition 6; Rat 2, Condition 1; Rat 3, Condition 6; Rat 10, Conditions 1 and 8). Having met all other stability criteria, the condition was terminated and, in the subsequent condition, the criterion was set equal to the mean percentage of left lever responses in the preceding condition but the position of the bonus lever was unchanged.

After at least four conditions, the session duration was increased or decreased for individual rats. This allowed for within-subject assessments of the effect of session duration on sensitivity to the bonus contingency. The three rats in the 15 -min group were given 60 -min sessions. Rats 4 and 9 were changed from 60-min to 15-min sessions. Rat 10 was given 120-min sessions after completing several conditions under 60-min sessions. 
Results

Table 1 shows the order of conditions and the stable data from the last nine sessions of each condition for each rat. Response rates on the left lever and right lever are shown separately. Percentage of left lever responses represents the left lever response rate divided by the sum of the left and right lever response rates multiplied by 100 . The average number of bonus pellets and the number of sessions in each condition are also shown. Values in parentheses are standard deviations. Figure 1 shows data from each rat in each condition. The shift toward the left lever relative to the criterion is shown. Positive values indicate a shift toward the left lever and negative values indicate a shift toward the right. The degree of shift is the difference between the mean percentage of left responses across the last nine sessions and the criterion. The solid horizontal line at zero represents the criterion value, the value produced if the mean percentage of left responses did not change relative to the criterion (the mean percentage of left responses in the preceding condition). Solid bars indicate that the bonus was on the left and shaded bars indicate that the bonus was on the right.

Shifts in responding did occur, however, for none of the rats were these shifts clearly a function of the bonus contingency. The magnitude of the shifts ranged from about 0 to 10 percentage points across all rats and conditions. For no rat did responding shift in the appropriate direction in all conditions, but for some rats, shifts were in the appropriate direction more often than not. For example, in all conditions except Conditions 5, 9, and 10, the responding of Rat 4 shifted in the direction of the bonus contingency. The responding of Rat 9 shifted in the inappropriate direction in the first two conditions but shifted in the appropriate direction across six of the last seven conditions. Rat 3 tracked the bonus contingency across the first three 
Table 1

Experiment 1. The order of conditions and the stable data from the last nine sessions of each are shown for each rat. The letters $L$ and $R$ in the condition label represent the position of the bonus contingency, left or right, respectively. The number in the condition label represents the session duration $(15,60$, or $120 \mathrm{~min})$. Response rates on the left lever and right lever are shown separately. Percentage of left lever responses (Percentage Left) represents the left lever response rate divided by the sum of the left and right lever response rates multiplied by 100 . The average number of bonus pellets and the number of sessions in each condition are also shown. Values in parentheses are standard deviations.

\begin{tabular}{|c|c|c|c|c|c|c|c|c|c|c|}
\hline \multirow{2}{*}{ Rat } & \multirow{2}{*}{$\begin{array}{c}\text { Condi- } \\
\text { tion }\end{array}$} & \multicolumn{4}{|c|}{ Responses per min } & \multirow{2}{*}{\multicolumn{2}{|c|}{ Percentage }} & \multirow[b]{2}{*}{ Bonus } & \multirow[b]{2}{*}{ Pellets } & \multirow[b]{2}{*}{ Sessions } \\
\hline & & \multicolumn{2}{|c|}{ Left } & \multicolumn{2}{|c|}{ Right } & & & & & \\
\hline 1 & L15 & 22.1 & $(1.9)$ & 32.9 & $(5.4)$ & 40.5 & $(3.0)$ & 2.4 & $(3.2)$ & 10 \\
\hline & R15 & 21.7 & $(2.8)$ & 33.3 & $(3.7)$ & 39.5 & $(3.8)$ & 3.6 & $(4.7)$ & 17 \\
\hline & L15 & 21.2 & $(3.6)$ & 34.8 & $(4.6)$ & 37.8 & $(4.3)$ & 1.8 & $(3.2)$ & 18 \\
\hline & R15 & 24.2 & $(2.5)$ & 35.2 & $(7.3)$ & 41.2 & $(3.8)$ & 0.9 & $(1.9)$ & 9 \\
\hline & L60 & 12.5 & $(1.8)$ & 22.1 & $(3.5)$ & 36.3 & $(3.2)$ & 0.2 & $(0.6)$ & 27 \\
\hline 2 & R15 & 32.9 & $(2.4)$ & 17.6 & $(2.5)$ & 65.2 & $(3.8)$ & 0.0 & $(0.0)$ & 9 \\
\hline & R15 & 34.4 & $(3.0)$ & 18.1 & $(2.1)$ & 65.5 & $(3.5)$ & 2.4 & $(3.7)$ & 10 \\
\hline & L15 & 39.0 & $(4.5)$ & 17.7 & $(1.6)$ & 68.6 & $(1.9)$ & 2.4 & $(4.6)$ & 14 \\
\hline & R15 & 41.8 & $(2.6)$ & 18.1 & $(1.6)$ & 69.8 & $(2.2)$ & 0.7 & $(1.9)$ & 16 \\
\hline & L15 & 31.7 & $(4.8)$ & 15.7 & $(2.3)$ & 66.7 & $(3.7)$ & 1.1 & $(3.1)$ & 19 \\
\hline & $\mathrm{R} 60$ & 35.0 & $(3.0)$ & 19.9 & $(1.6)$ & 63.7 & $(3.2)$ & 5.8 & $(5.2)$ & 11 \\
\hline & L 60 & 30.2 & $(4.4)$ & 14.3 & $(1.7)$ & 68.1 & $(4.6)$ & 8.7 & $(8.4)$ & 30 \\
\hline & $\mathrm{R} 60$ & 29.3 & $(6.5)$ & 20.3 & $(3.6)$ & 60.2 & $(3.4)$ & 14.0 & $(5.3)$ & 13 \\
\hline & L 60 & 19.9 & $(7.1)$ & 15.2 & $(4.0)$ & 56.1 & $(3.5)$ & 0.0 & $(0.0)$ & 18 \\
\hline & $\mathrm{R} 60$ & 16.6 & $(4.7)$ & 12.4 & $(3.2)$ & 57.3 & $(4.4)$ & 2.0 & $(2.7)$ & 12 \\
\hline 3 & R60 & 44.1 & $(3.7)$ & 33.8 & $(2.8)$ & 56.6 & $(2.9)$ & 8.9 & $(5.7)$ & 9 \\
\hline & L 60 & 54.3 & $(2.6)$ & 30.2 & $(2.8)$ & 64.3 & $(2.9)$ & 14.2 & $(6.1)$ & 15 \\
\hline & R60 & 48.3 & $(1.8)$ & 31.9 & $(2.5)$ & 60.3 & $(2.3)$ & 7.1 & $(4.7)$ & 17 \\
\hline & L 60 & 46.2 & $(2.2)$ & 34.4 & $(3.0)$ & 57.4 & $(2.3)$ & 0.2 & $(0.6)$ & 18 \\
\hline & $\mathrm{R} 60$ & 45.6 & $(3.9)$ & 27.5 & $(3.3)$ & 62.4 & $(3.6)$ & 0.2 & $(0.6)$ & 13 \\
\hline & L 60 & 36.8 & $(3.9)$ & 30.2 & $(3.7)$ & 55.0 & $(2.8)$ & 0.0 & $(0.0)$ & 17 \\
\hline 4 & R60 & 31.5 & $(3.3)$ & 31.7 & $(3.7)$ & 49.9 & $(2.2)$ & 12.2 & $(4.4)$ & 9 \\
\hline & L 60 & 36.4 & $(4.1)$ & 30.0 & $(2.3)$ & 54.8 & $(3.4)$ & 9.3 & $(6.3)$ & 9 \\
\hline & $\mathrm{R} 60$ & 27.4 & $(2.5)$ & 32.6 & $(2.9)$ & 45.7 & $(1.6)$ & 15.6 & $(3.2)$ & 24 \\
\hline & L 60 & 33.8 & $(2.5)$ & 33.5 & $(3.6)$ & 49.8 & $(3.0)$ & 7.1 & $(6.1)$ & 11 \\
\hline & R15 & 35.5 & $(3.8)$ & 32.3 & $(3.6)$ & 51.7 & $(2.2)$ & 0.4 & $(1.3)$ & 13 \\
\hline & L15 & 34.6 & $(5.8)$ & 26.5 & $(6.0)$ & 56.9 & $(4.8)$ & 10.2 & $(9.1)$ & 30 \\
\hline & R15 & 37.4 & $(3.1)$ & 35.2 & $(3.5)$ & 51.6 & $(2.2)$ & 10.0 & $(4.3)$ & 9 \\
\hline & L15 & 30.3 & $(4.1)$ & 24.5 & $(3.4)$ & 55.2 & $(3.4)$ & 6.7 & $(6.2)$ & 22 \\
\hline & R15 & 21.3 & $(5.0)$ & 13.9 & $(2.0)$ & 60.0 & $(3.8)$ & 0.2 & $(0.6)$ & 29 \\
\hline & L15 & 19.7 & $(1.9)$ & 13.7 & $(1.6)$ & 59.0 & $(2.7)$ & 1.1 & $(2.5)$ & 10 \\
\hline 8 & R15 & 33.5 & $(2.7)$ & 18.6 & $(2.6)$ & 64.5 & $(2.0)$ & 1.1 & $(2.1)$ & 9 \\
\hline & L15 & 33.0 & $(3.5)$ & 18.7 & $(2.6)$ & 63.8 & $(3.4)$ & 2.0 & $(3.0)$ & 9 \\
\hline & R15 & 35.5 & $(3.0)$ & 21.4 & $(2.5)$ & 62.5 & $(2.2)$ & 1.8 & $(3.2)$ & 18 \\
\hline & L15 & 33.8 & $(3.4)$ & 22.2 & $(2.0)$ & 60.3 & $(2.8)$ & 0.7 & $(1.3)$ & 9 \\
\hline & $\mathrm{R} 60$ & 24.6 & $(4.4)$ & 20.6 & $(2.9)$ & 54.3 & $(3.0)$ & 11.1 & $(5.7)$ & 11 \\
\hline & L 60 & 20.0 & $(2.4)$ & 20.0 & $(3.4)$ & 50.1 & $(2.6)$ & 0.2 & $(0.6)$ & 31 \\
\hline & $\mathrm{R} 60$ & 16.6 & $(0.7)$ & 17.6 & $(2.0)$ & 48.7 & $(2.5)$ & 2.7 & $(4.1)$ & 14 \\
\hline
\end{tabular}


Table 1 (continued)

\begin{tabular}{|c|c|c|c|c|c|c|c|c|c|c|}
\hline \multirow[b]{2}{*}{ Rat } & \multirow{2}{*}{$\begin{array}{l}\text { Condi- } \\
\text { tion }\end{array}$} & \multicolumn{4}{|c|}{ Responses per min } & \multirow{2}{*}{\multicolumn{2}{|c|}{ Percentage Left }} & \multirow[b]{2}{*}{ Bonus } & \multirow[b]{2}{*}{ Pellets } & \multirow[b]{2}{*}{ Sessions } \\
\hline & & \multicolumn{2}{|c|}{ Left } & \multicolumn{2}{|c|}{ Right } & & & & & \\
\hline 9 & R60 & 39.4 & $(3.8)$ & 21.3 & $(1.6)$ & 64.9 & $(3.1)$ & 0.0 & $(0.0)$ & 17 \\
\hline & $\mathrm{L} 60$ & 34.8 & $(2.8)$ & 20.7 & $(2.2)$ & 62.7 & $(3.2)$ & 0.9 & $(1.9)$ & 10 \\
\hline & $\mathrm{R} 60$ & 30.9 & $(3.6)$ & 22.2 & $(1.5)$ & 58.1 & $(3.1)$ & 8.2 & $(6.1)$ & 17 \\
\hline & $\mathrm{L} 60$ & 33.1 & $(4.3)$ & 18.5 & $(2.0)$ & 64.0 & $(3.3)$ & 11.1 & $(6.7)$ & 15 \\
\hline & $\mathrm{R} 60$ & 28.6 & $(4.5)$ & 19.0 & $(2.5)$ & 60.0 & $(3.4)$ & 7.8 & $(5.8)$ & 9 \\
\hline & L 60 & 42.1 & $(3.2)$ & 18.4 & $(2.0)$ & 69.6 & $(2.8)$ & 18.4 & $(5.4)$ & 12 \\
\hline & R15 & 33.2 & $(6.0)$ & 18.9 & $(2.9)$ & 63.6 & $(3.5)$ & 11.1 & $(6.4)$ & 16 \\
\hline & L15 & 34.4 & $(4.3)$ & 15.0 & $(2.7)$ & 69.7 & $(4.0)$ & 11.1 & $(7.5)$ & 21 \\
\hline & R15 & 32.5 & $(3.5)$ & 12.0 & $(3.5)$ & 73.2 & $(6.5)$ & 1.8 & $(2.9)$ & 18 \\
\hline 10 & $R 60$ & 38.5 & $(3.9)$ & 26.7 & $(2.8)$ & 59.0 & $(2.8)$ & 0.4 & $(1.3)$ & 9 \\
\hline & R60 & 35.8 & $(3.2)$ & 24.0 & $(2.0)$ & 59.8 & $(3.3)$ & 1.3 & $(3.1)$ & 9 \\
\hline & L 60 & 29.3 & $(2.4)$ & 21.6 & $(1.4)$ & 57.5 & $(2.7)$ & 0.9 & $(1.7)$ & 10 \\
\hline & $\mathrm{R} 60$ & 28.4 & $(3.0)$ & 28.0 & $(3.6)$ & 50.4 & $(3.1)$ & 13.3 & $(6.5)$ & 12 \\
\hline & L 60 & 27.9 & $(2.9)$ & 25.7 & $(3.8)$ & 52.2 & $(4.2)$ & 4.0 & $(7.5)$ & 13 \\
\hline & R120 & 19.7 & $(2.8)$ & 18.0 & $(2.7)$ & 52.3 & $(2.0)$ & 1.3 & $(1.6)$ & 12 \\
\hline & L120 & 19.3 & $(2.6)$ & 19.1 & $(3.1)$ & 50.4 & $(2.9)$ & 0.7 & $(1.3)$ & 13 \\
\hline & R120 & 19.4 & $(1.5)$ & 18.6 & $(1.4)$ & 51.1 & $(1.5)$ & 0.2 & $(0.6)$ & 19 \\
\hline & L120 & 13.9 & $(1.0)$ & 15.0 & $(1.3)$ & 48.0 & $(2.7)$ & 0.2 & $(0.6)$ & 9 \\
\hline
\end{tabular}


Next Page

Figure 1. Experiment 1. Percentage shift in responding in each condition. Above each condition is a letter. An "L" represents conditions in which the bonus contingency was on the left lever and an " $\mathrm{R}$ " indicates that the bonus contingency was on the right lever. The data are represented by filled bars when the bonus contingency was on the left lever and by shaded bars when the bonus contingency was on the right lever. An upward shift indicates an increase in the percentage of left lever responses and a downward shift indicates a decrease in the percentage of left lever responses. The number above a particular condition indicates the session duration, in minutes. 


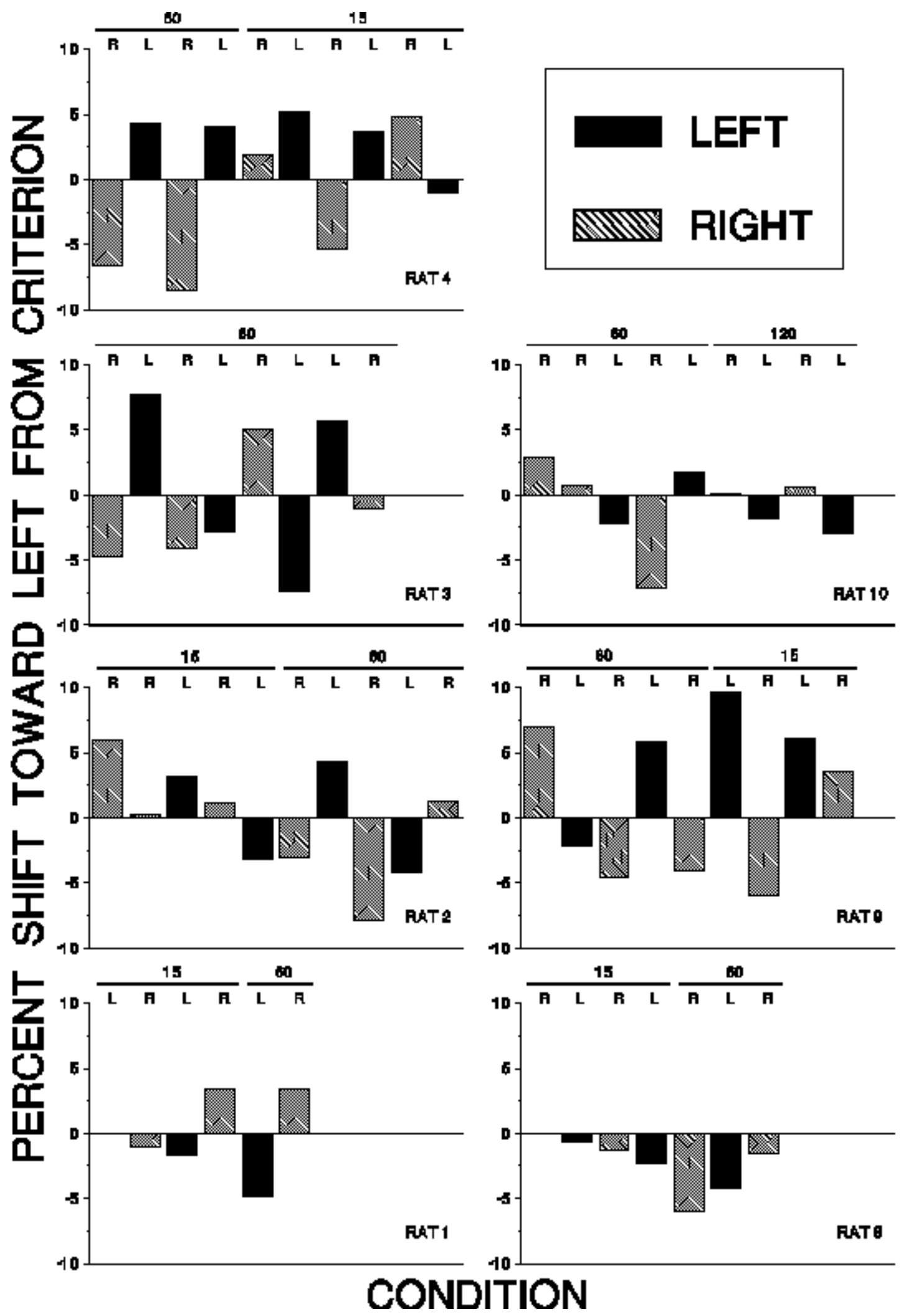


conditions, however, in the last five conditions responding shifted appropriately only twice. Taken together, the performances of these three rats provide no conclusive evidence of control by the molar contingency.

The other four rats showed no evidence of sensitivity to the molar contingency. For example, the responding of Rat 10 shifted in the appropriate direction in only two of nine conditions, poorer performance than expected by chance alone. Similarly, the responding of Rat 1 shifted in the appropriate direction in only two of five conditions and for Rat 2 appropriate shifts occurred in only four of ten conditions. Perhaps more informative is the performance of Rat 8. This responding of this rat shifted toward the right lever across each condition, regardless of the position of the bonus contingency. This resulted in appropriate shifts in four of seven conditions (the four conditions in which the bonus was on the right lever). For Rat 8 , the mean percentage of left lever responses began near 65 in Condition 1 and decreased in each subsequent condition to a terminal value of about 50. Thus, Rat 8 showed adaptation to the concurrent schedule arrangement as bias toward the left lever decreased with continued exposure until both levers were chosen equally often. However, this result suggests no control of choice responding by the molar contingency. In sum, the distribution of responses on the two levers was poorly controlled by the molar bonus contingency. Even for rats whose behavior often shifted appropriately with shifts in the location of the bonus contingency, control by the molar contingency overall was neither robust nor reliable.

An effect of session duration was not apparent. In general, rats in the 60-min group seemed to track the molar contingency more so than rats in the 15-min group. However, due to the small number of rats in the two groups, this small difference may be due to sampling bias. 
Also, within-subject comparisons of different session durations revealed no apparent differences. Increasing the session duration from 15 to $60 \mathrm{~min}$ did not appear to alter control by the bonus contingency, with the possible exception of Rat 2 . For Rat 10, increasing the session duration from 60 to $120 \mathrm{~min}$ had no obvious effects on behavior. For Rats 4 and 9 (the two rats showing the greatest proportion of appropriate shifts), decreasing the session duration from 60 to $15 \mathrm{~min}$ did not systematically affect performance. In sum, manipulations of session duration had no consistent effect on control by the molar contingency.

Figure 2 shows the number of bonus pellets earned in each session for each rat across all experimental conditions. The rats did come into contact with the molar contingency, but sessionto-session variability in the number of bonus pellets earned was high. Additionally, when choice shifted in the direction of the bonus lever and produced bonus pellets, continued change in choice in the direction of the bonus lever was slow to occur, if at all. Thus, the molar contingency did not have immediate or unambiguous effects on choice. Overall, contact with the bonus pellets was infrequent, occurring in a majority of sessions for only two rats (Rats 4 and 9).

\section{Discussion}

The results of the present experiment were similar to those of Mellitz et al. (1983). Shifts in responding occurred for all rats, but not even a majority of rats showed control by the molar contingency. As in Mellitz et al.'s study, some suggestive, but not conclusive, evidence of control was observed for two rats. The present experiment provided a more stringent test of control, however. First, more reversals were conducted than by Mellitz et al. and this allowed a stronger determination of the reliability of control. The responding of not even one rat shifted appropriately in all conditions, and across subjects, the number of appropriate shifts was close to 


\section{Next Page}

Figure 2. Experiment 1. Number of bonus pellets for each session of each experimental condition. The data are presented individually for each rat. The position of the bonus contingency (L, left, or $\mathrm{R}$, right) and the session duration (15, 60, or $120 \mathrm{~min})$ appear at the top of each condition. 


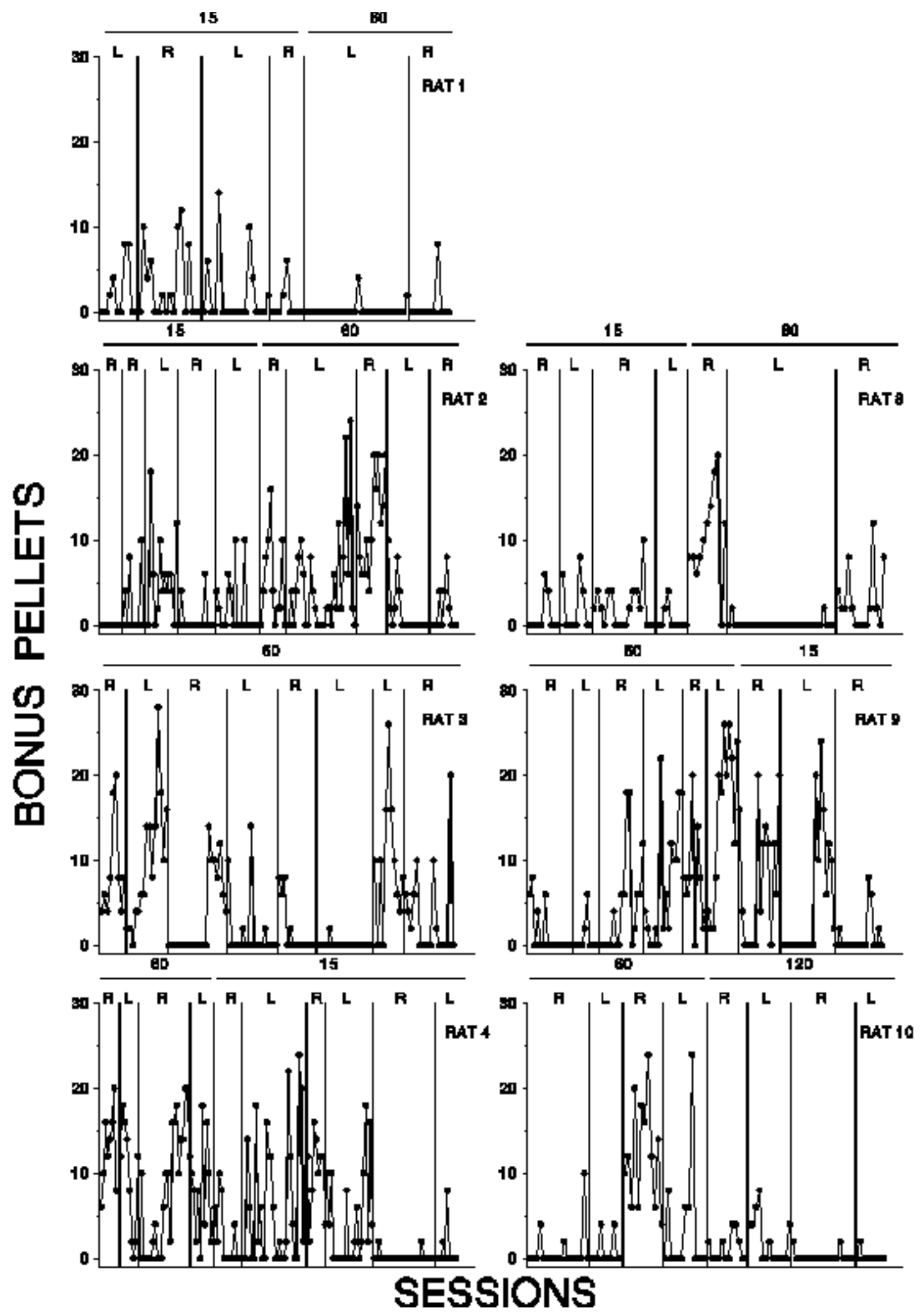


that predicted by chance alone. Had only four conditions been conducted, the behavior of Rat 4 would have seemed incredibly sensitive to the bonus contingency. That shifts were inappropriate in later reversals suggests that molar control did not dissipate with experience, but rather, such control was never established. Further, the use of a nine-session stability criterion ensured that transient shifts over a few sessions would not represent the choice data from a particular condition. Recall that Mellitz et al. used a three-session criterion.

It is arguable that control of choice was not established in the present experiment because the interdependent VI schedules constrained the degree of shift possible. In Mellitz et al., exclusive preference for one lever would not reduce reinforcement rate as in the present study. In fact, shifts in choice were sometimes quite large in their study. However, sizable and detectable shifts were observed in the present study. Certainly, shifts in choice were not prevented by the concurrent VI VI schedule. Why these shifts occurred is not obvious given the absence of clear control by the bonus contingency.

It is possible that Mellitz et al. (1983) arranged a more effective molar consequence: reduced contact with a schedule of shock postponement. One can conceive of their sessionduration reduction contingency as a delayed escape contingency. Responses on the conjoint lever avoided shocks in the short run and escaped shocks in the long run. Thus, the delayed reinforcer (escape) was qualitatively different from the more immediate reinforcer (avoidance). It is likely that escape functions as a stronger reinforcer than avoidance, particularly under unsignaled avoidance procedures, because escape produces a clear safety signal that should reinforce responses that produce it. In the present study, the delayed and immediate reinforcers were qualitatively similar (i.e., food pellets). Perhaps a delayed reinforcer that is qualitatively different 
from and preferred over the reinforcer earned immediately would be more likely to establish control by molar response-reinforcer relations. However, the similarity of our results to those of Mellitz et al. suggest that this may not be an extremely important variable. Aggregate responsereinforcer relations, on the order of $15 \mathrm{~min}$ or more, do not appear to control choice reliably.

\section{Experiment 2}

In Experiment 2, control of aggregated responses by a delayed consequence was assessed using a procedure that did not involve choice. A schedule of intermittent, but immediate, reinforcement operated throughout the session. During discrete periods signaled by a visual stimulus, responding also counted toward a small ratio requirement, the completion of which initiated an unsignaled delay to a large reinforcer. Responding should be higher in the presence of the stimulus than in its absence when the delayed reinforcer is controlling responding. The degree of response elevation serves as an index of the degree of control by the large, delayed reinforcer.

The procedure was modeled after that used by DeWaard, Galizio, and Baron (1979), but used positive rather than negative reinforcement. The duration of the delay to the large reinforcer was manipulated across conditions in a steady-state fashion. The goal was to determine at which delays response rate during the stimulus would be reliably higher than response rates during the VI schedule immediately before the stimulus onset, thereby suggesting control of behavior by the delayed reinforcement contingency.

Unlike Experiment 1, this procedure ensured contact with the delayed consequences of responding. In Experiment 1, the molar consequence was encountered at most once per session and, on average, less than once every two sessions (Figure 2). Failure to convincingly establish 
molar control may have been due to infrequent contact between responding and the molar consequence. Experiment 2 avoided this complication by making the molar consequence contingent on a short sequence of responses that was completed several times per session. Under conditions in which the molar consequence did not control higher rates of responding, the response requirement was completed nevertheless. This design feature ensured frequent contact with the delayed consequence and was intended to facilitate control by the molar responsereinforcer relation. Furthermore, elevated responding was established relatively early in the experiment at short delays. Subsequent conditions arranged progressively longer delays until the degree of control by the bonus was small (response rates during the stimulus approximated baseline VI rates). Thus, a demonstration of control at short delays followed by the elimination or reduction of such control at longer delays provides strong evidence for the role of the delay variable in controlling elevated responding.

\section{Method}

\section{$\underline{\text { Subjects and Apparatus }}$}

Four experimentally naive male Sprague-Dawley rats were used. The rats were about 90 days old at the start of the experiment and were maintained at approximately $80 \%$ of free-feeding weight via daily post-session feedings given at least $30 \mathrm{~min}$ after being returned to their home cages. The apparatus was the same as in Experiment 1, except for the addition of a water bottle mounted on the outside of each operant chamber. The spout of the bottle protruded approximately $2.5 \mathrm{~cm}$ into the back wall of the chamber and was about $4 \mathrm{~cm}$ above the grid floor. 


\section{$\underline{\text { Procedure }}$}

Preliminary training. Each rat was taught to approach the food tray and promptly consume the pellet upon delivery. Pressing the right lever was established by reinforcing successive approximations to this behavior. After 100 reinforced lever presses the session was terminated. During shaping, only the houselight provided illumination. Although the left lever was not used in the experiment, both levers were present throughout the experiment. Few responses occurred to the left lever throughout the course of the study.

The goal of preliminary training was to maintain lever pressing under a VI 150-s schedule. Each session, during which the houselight was on, ended after 90 min or 100 pellets, whichever came first. The mean interval of the VI schedule was increased across sessions from 15 to $30,60,90$, and $150 \mathrm{~s}$. Each VI schedule consisted of 12 unique intervals. The intervals were distributed to approximate a random-probability distribution, as described by Fleshler and Hoffman (1962). The 12 intervals were arranged in three random orders to produce a list of 36 intervals. At the beginning of each session, a random starting point was selected. The VI 150-s schedule remained in effect for a minimum of 5 sessions, until the reliability of lever pressing was apparent and showed no decreasing trend by visual inspection of the graphed response rates.

Experimental conditions. A chained schedule was superimposed upon the VI 150-s schedule at irregular intervals throughout each session. The onset of the initial link occurred independently of responding. The initial links were presented 4, 6 , or 8 min after the onset of the session or from the end of the preceding terminal link (the point at which delivery of the bonus was completed).

The initial link was accompanied by illumination of the right cue light. The VI 150-s 
schedule continued to operate during the initial link. Fifteen responses completed the initial link (a fixed-ratio, FR, 15 schedule). Completion of the initial link turned off the right cue light and began the terminal link. The stimulus during the terminal link was identical to that during the VI 150-s schedule (houselight on). The first portion of the terminal link was a delay during which the VI schedule continued to operate. At the end of the delay, six bonus pellets were delivered at a rate of 1 per s. The VI schedule was suspended only during delivery of the bonus pellets. After the sixth bonus pellet was delivered, the VI schedule was reinstated. A minimum of 4 min of VI preceded the next presentation of the chained schedule. The response rate during the 3-min period before the onset of each initial link provided a baseline under the VI 150-s schedule. In each session, the chained schedule was presented 12 times. The session ended after delivery of the twelfth bonus.

Order of conditions. The order of conditions and the number of sessions in each are shown in Table 2. The conditions differed only in the delay to the bonus pellets in the terminal link. The exact delays used were 0.01 (hereafter referred to as 0), 10, 20, 40, 80 and $160 \mathrm{~s}$. Each delay condition remained in effect until performance met stability criteria (see Stability Criteria below). For two rats, the order of the first four conditions was 40, 0, 10, and $20 \mathrm{~s}$. For the remaining two rats, the order was 40,20,10, and $0 \mathrm{~s}$. After these four conditions were completed, all rats were exposed next to the 80-s condition and, for three rats, this was followed by the 160-s condition. Finally, the original 40-s condition was replicated for all rats.

Stability criteria. Each condition was in effect for a minimum of 20 sessions. Stable performance was based upon the last six sessions. For each session, the mean response rates of the 12 initial links and of the twelve baseline periods were determined. For performance to be 
Table 2

Order of conditions, the number of sessions in each, and the median and interquartile ranges of baseline, initial-link, and terminal-link response rates for each condition. Also shown are the median and interquartile range of difference scores (initial-link minus baseline responses per min) in each condition. An asterisk next to the difference score indicates that the result of a one-tailed sign test of the difference scores was significantly greater than zero in that condition.

\begin{tabular}{|c|c|c|c|c|c|c|c|c|c|c|}
\hline \multirow{3}{*}{$\frac{\text { Rat }}{\mathrm{C} 1}$} & \multirow{3}{*}{$\frac{\text { Delay }}{40}$} & \multirow{3}{*}{$\begin{array}{l}\text { Sess- } \\
\frac{\text { ions }}{20}\end{array}$} & \multicolumn{8}{|c|}{ Responses per Min } \\
\hline & & & \multicolumn{2}{|c|}{ Baseline } & \multicolumn{2}{|c|}{ Initial-Link } & \multicolumn{2}{|c|}{ Terminal-Link } & \multicolumn{2}{|c|}{ Difference Score } \\
\hline & & & 7.0 & $(4.0-8.0)$ & 7.5 & $(6.5-9.6)$ & 6.0 & $(6.0-7.5)$ & 1.7 & $(-0.3$ to 4.4$) *$ \\
\hline & 20 & 35 & 11.5 & $(9.0-17.0)$ & 24.0 & $(18.3-31.1)$ & 9.0 & $(6.0-9.0)$ & 10.3 & $(5.5$ to 16.8$)$ * \\
\hline & 10 & 27 & 13.5 & $(9.0-17.0)$ & 30.5 & $(23.2-36.2)$ & 0.0 & $(0.0-6.0)$ & 15.6 & $(10.5$ to 24.0$)$ * \\
\hline & 0 & 28 & 14.0 & $(11.0-19.0)$ & 85.1 & $(73.4-98.3)$ & & & 70.6 & $(60.0$ to 86.6$)$ * \\
\hline & 80 & 30 & 10.0 & $(7.0-11.0)$ & 17.1 & $(12.4-22.3)$ & 10.5 & $(8.3-12 \cdot 0)$ & 8.5 & $(3.1$ to 13.9$) *$ \\
\hline & 160 & 20 & 9.0 & $(7.0-10.0)$ & 11.9 & $(10.1-15.1)$ & 9.4 & $(8.3-10.5)$ & 4.2 & $(2.0$ to 6.3$) *$ \\
\hline & 40 & 20 & 9.0 & $(7.0-12.0)$ & 23.7 & $(15.6-29.9)$ & 7.5 & $(6.0-9.0)$ & 12.6 & $(7.3$ to 20.4$)$ * \\
\hline \multirow[t]{7}{*}{ C4 } & 40 & 24 & 16.0 & $(12.0-20.0)$ & 25.5 & $(19.9-32.5)$ & 6.0 & $(4.5-9.0)$ & 8.7 & $(3.1$ to 15.5$)$ * \\
\hline & 20 & 26 & 29.0 & $(25.0-35.0)$ & 62.7 & $(53.0-72.7)$ & 6.0 & $(3.0-12 \cdot 0)$ & 30.6 & $(23.4$ to 42.2$)$ * \\
\hline & 10 & 30 & 32.0 & $(27.0-37.0)$ & 70.1 & $(57.8-83.0)$ & 6.0 & $(0.0-6.0)$ & 37.5 & $(23.5$ to 50.4$)$ * \\
\hline & 0 & 20 & 38.0 & $(31.0-43.0)$ & 96.4 & $(77.1-110.2)$ & & & 57.4 & $(37.0$ to 79.0$)$ * \\
\hline & 80 & 39 & 26.0 & $(17.0-35.0)$ & 43.6 & $(35.0-59.1)$ & 19.5 & $(16.5-24.8)$ & 19.0 & $(10.7$ to 26.8$)$ * \\
\hline & 160 & 20 & 20.0 & $(14.0-29.0)$ & 25.8 & $(18.6-36.6)$ & 21.2 & $(18.0-24.8)$ & 5.0 & $(-1.7$ to 14.3$)$ * \\
\hline & 40 & 22 & 29.5 & $(22.0-38.0)$ & 49.6 & $(37.7-69.9)$ & 13.5 & $(10.5-18.0)$ & 18.6 & $(10.9$ to 35.7$)$ * \\
\hline \multirow[t]{7}{*}{ C7 } & 40 & 20 & 15.0 & $(12.0-17.0)$ & 16.1 & $(13.0-19.5)$ & 13.5 & $(10.5-16.5)$ & 1.4 & $(-2.5$ to 4.5$) *$ \\
\hline & 0 & 29 & 43.0 & $(37.0-50.0)$ & 90.2 & $(77.2-106.4)$ & & & 48.3 & $(32.8$ to 62.0$)$ * \\
\hline & 10 & 36 & 46.0 & $(36.0-56.0)$ & 68.1 & $(51.5-78.6)$ & 66.0 & $(54.0-78.0)$ & 23.9 & $(1.7$ to 39.6$) *$ \\
\hline & 20 & 22 & 38.0 & $(28.0-46.0)$ & 63.2 & $(46.1-82.0)$ & 42.0 & $(27.0-51.0)$ & 23.3 & $(9.3$ to 43.4$)$ * \\
\hline & 80 & 38 & 26.5 & $(17.0-32.0)$ & 34.1 & $(25.2-42.9)$ & 27.8 & $(21.8-33.8)$ & 6.5 & (1.4 to 18.6$) *$ \\
\hline & 160 & 22 & 19.0 & $(12.0-23.0)$ & 24.1 & $(18.5-32.7)$ & 21.0 & $(18.4-23.6)$ & 7.2 & $(-0.1$ to 14.6$)$ * \\
\hline & 40 & 21 & 15.0 & $(12.0-19.0)$ & 17.3 & $(11.7-25.5)$ & 13.5 & $(10.5-15.0)$ & 2.3 & $(-2.8$ to 5.9$)$ \\
\hline \multirow[t]{6}{*}{$\mathrm{C} 10$} & 40 & 21 & 17.0 & $(11.0-21.0)$ & 22.8 & $(19.2-26.1)$ & 22.5 & $(19.5-28.5)$ & 6.5 & $(0.9$ to 11.2$)$ * \\
\hline & 0 & 34 & 34.0 & $(26.0-42.0)$ & 67.6 & $(47.5-78.0)$ & & & 30.1 & $(15.3$ to 43.4$)$ * \\
\hline & 10 & 33 & 37.0 & $(24.0-47.0)$ & 52.3 & $(34.0-63.8)$ & 27.0 & $(18.0-30.0)$ & 16.4 & $(-0.1$ to 29.8$)$ * \\
\hline & 20 & 21 & 47.5 & $(28.0-57.0)$ & 45.9 & $(19.8-66.8)$ & 24.0 & $(15.0-33.0)$ & 4.7 & $(-20.5$ to 27.2$)$ \\
\hline & 80 & 35 & 42.0 & $(23.0-58.0)$ & 39.5 & $(20.6-60.1)$ & 52.5 & $(37.5-66.0)$ & 5.0 & $(-18.9$ to 18.1$)$ \\
\hline & 40 & 23 & 26.5 & $(13.0-43.0)$ & 30.5 & $(16.0-44.5)$ & 33.8 & $(22.5-45.0)$ & 4.9 & $(-7.3$ to 14.7$)$ \\
\hline
\end{tabular}


considered stable, both the initial-link and baseline response rates had to meet a mathematical stability criterion. The mean of the first and last three sessions had to be within $10 \%$ of the grand mean of the last six sessions.

\section{Results}

Although the stability criteria were based on mean response rates, considerable variability in these response rates within the session was observed, and the distributions of response rate were often positively skewed, particularly for initial-link response rate. For this reason, the analyses presented below are based on medians and interquartile ranges, which are more appropriate measures of central tendency and variability for skewed distributions.

Recall that the baseline period was $3 \mathrm{~min}$ in duration and that the time from the delivery of the bonus to the start of the next baseline period varied from 1 to $5 \mathrm{~min}$. Two observations suggested that the baseline period should be shortened to the final $1 \mathrm{~min}$ prior to initial-link onset. First, responding was noticeably suppressed following the bonus for all rats and occasionally this period of suppression was included in the early portion of the baseline period. Second, responding was usually higher during the final portion of the baseline period than during the early portion. Thus, the use of the 3-min baseline was inappropriate for assessing sensitivity to the initial-link contingency because it underestimated prevailing baseline response rates. Furthermore, because sensitivity in the procedure is indexed by the local change in response rate produced by the onset of the initial link and its correlated stimulus, a more sensitive assessment of local response rate changes would be to examine only the last $1 \mathrm{~min}$ of the baseline period for the purposes of calculating response rate. For these reasons, the baseline response rates reported herein were based on the final 1 min of each baseline period. 
Table 2 shows the order of conditions, the number of sessions in each, and the median and interquartile ranges of the baseline, initial-link, and terminal-link response rates for each condition. In the 0-s condition, no terminal-link responses could occur so terminal-link response rate is not shown. Figure 3 shows the response rate data in graphic form. The median baseline, initial-link, and terminal-link response rates are shown for each delay value studied. The data from the replication of the 40-s condition are indicated by empty symbols. For purposes of drawing the line function, the mean of both median response rates from the 40-s conditions was used.

Initial-link response rates were higher than baseline response rates in all conditions. In general, initial-link response rates increased with decreased delays to the bonus. Note that baseline response rates also tended to increase with decreased delays, but to a lesser extent than initial-link rates. Changes in terminal-link response rate were less orderly and varied between subjects. For Rats $\mathrm{C} 1, \mathrm{C} 4$, and $\mathrm{C} 10$, terminal-link response rates increased with increased delays to the bonus, but generally decreased with with increased delays for Rat C7. For Rats C1 and C4, terminal-link response rates were similar to or lower than baseline and initial-link response rates in all conditions. For Rat C7, terminal-link rates sometimes exceeded baseline response rates, and for Rat C10 terminal-link rates were higher than baseline and initial-link rates at the longest delays (40 and $80 \mathrm{~s}$ ). This latter pattern exhibited by Rat C10 suggests generalized acceleration of responding with the passage of time since the last bonus, rather than control by the initial-link stimulus. Indeed, the similar baseline and initial-link response rates at the longest delays seem to corroborate this interpretation. For three rats, response rates in the 3-min baseline were slightly but consistently lower than those based on the final 1 min only, but for Rat C10, the difference 

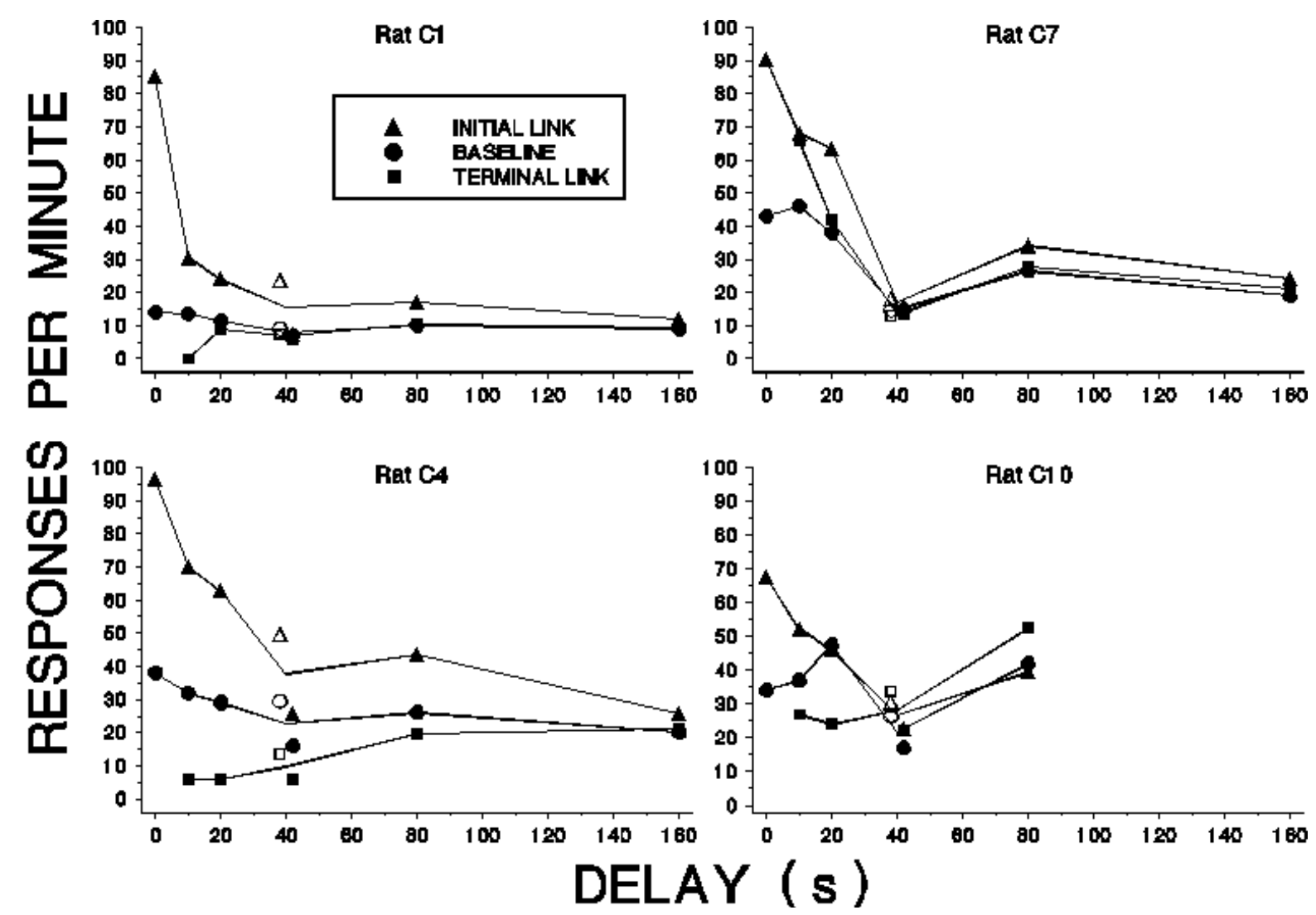

Figure 3. Experiment 2. Median baseline, initial-link, and terminal-link response rates, in responses per min, for each rat in each delay condition. Circles represent baseline, triangles represent initial link, and squares represent terminal link. The empty symbols represent data from the replication of the 40-s condition. The line functions connect the medians from each delay condition, except for $40 \mathrm{~s}$, in which the mean of both median response rates was used. 
was much larger. All of these observations suggest accelerated responding with the passage of time and poor control by the delayed reinforcer for Rat C10.

As mentioned previously, both baseline and initial-link responding increased with decreased delays, suggesting a correlation between the two. Figure 4 shows the baseline response rate as a function of the initial-link response rate, using the median baseline and initial-link responses per min from each condition. The data are graphed on log-log axes. A least-squares regression line was fit to the data of each rat and the corresponding r-squared value is shown in the graph. The dotted line in each panel represents a slope of 1.0, which would indicate that initial-link and baseline response rates changed similarly across conditions. For all rats, the slope of the line was positive but less than 1.0, indicating that baseline responding changed less than did initial-link responding with changes in delay. The regression lines describe the data well, except for Rat C10. The moderate r-squared value for Rat $\mathrm{C} 1$ reflects little variation in baseline response rate for which to account, rather than a poor linear fit of the data. It is clear that baseline and initial-link response rates were positively correlated for all rats. For this reason, proportional measures of initial-link enhancement (e.g., initial-link divided by baseline) were not appropriate. Because small changes in baseline response rate would produce large changes in such a measure, changes in baseline rate compromise the utility of such a measure. If initial-link response rates did not change but baseline response rates did, the proportional measure would change greatly, particularly when absolute baseline response rates are quite low (as in the case of Rat C1). Also, if baseline and initial-link response rates both increased, the change might not be detected by the proportional measure. For example, if baseline response rate was 10 responses per min and initial link was 50 responses per min, the index would equal 5.0, indicating a fivefold difference. If 


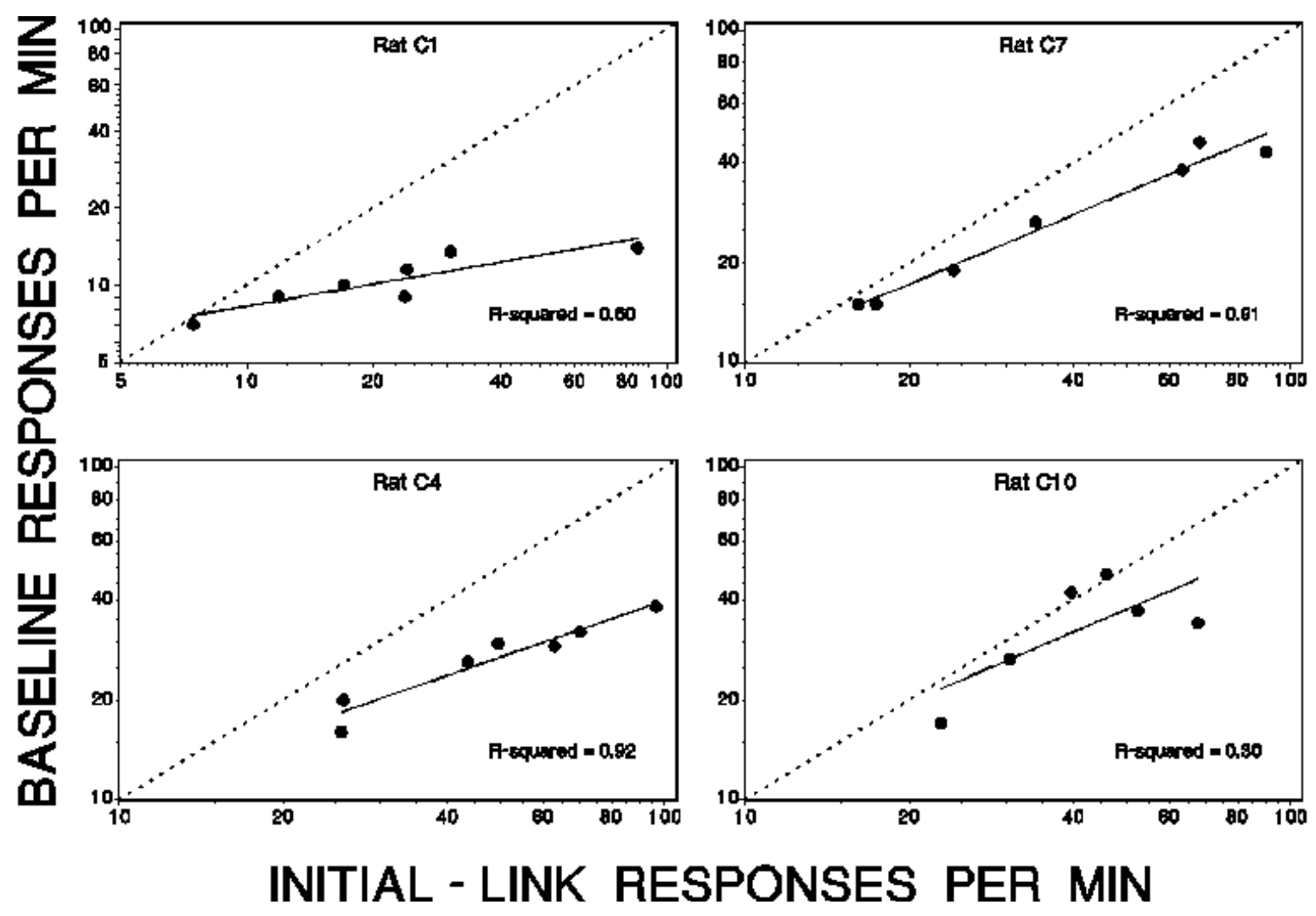

Figure 4. Experiment 2. Baseline responses per min as a function of initial-link responses per min for each rat. The data are plotted on log-log axes. The least-squares regression line is indicated by a solid line. The corresponding r-squared value is shown in the lower right of each panel. A slope of 1.0 is indicated by the dashed diagonal line. 
baseline response rate was 20 responses per min and initial link was 100 responses per min, the index would also equal 5.0, failing to reveal the increased absolute disparity between the two response rates. This latter point applies equally well to a commonly used proportional measure, the discrimination ratio, which in the present case would equal the initial-link response rate divided by the sum of the baseline and initial-link response rates. The discrimination ratio would be a poor index of control in the present procedure because the end points, 0 and 1.0, would not be anticipated given the experimental contingencies. Specifically, neither baseline nor initial-link response rates should ever equal 0 . For all of the above reasons, initial-link enhancement was calculated using a subtractive, rather than a proportional, measure. A difference score was calculated for each transition from baseline to initial link. The baseline response rate (based on the final $1 \mathrm{~min}$ of each baseline period) was subtracted from the initial-link response rate to yield a measure of initial-link elevation in responses per min. Unlike a proportional measure such as the one above, this subtractive measure fails to provide a common metric for between-subjects comparisons when absolute response rates differ across rats. However, the present experiment relied on within-subjects assessments of control by delayed reinforcement, making this a trivial drawback.

Because there were 12 transitions from baseline to initial link per session and because assessments were based on the last 6 sessions of each condition, 72 difference scores were calculated for each condition. The difference score equaled the initial-link response rate minus the baseline response rate. The median and interquartile range of these difference scores was determined. With this measure, a value of 0 indicates no change in responding from baseline to initial-link. Negative scores indicate higher responding in baseline and positive scores indicate 
higher responding in the initial link. Note that the present calculation of difference scores captures local shifts in response rate from each adjacent baseline and initial-link pair, making this analysis more sensitive than a comparison based on average differences in baseline and initiallink response rates over the entire session.

Table 2 shows the median and interquartile range of difference scores for each rat in each condition. Because the difference scores were positive but close to 0 at the longer delays, statistical tests were conducted to determine the reliability of these small elevations in initial-link response rate. A one-tailed sign test was performed on the difference scores to determine whether these scores were significantly higher than a mean value of 0 , at a significance level of $\underline{p}<0.05$. The conditions in which the results were significant are indicated by an asterisk next to the median difference score in Table 2. All elevations were significant except for Rat C10 in the 20and 80-s conditions and in the replication of the 40-s condition and for Rat C7 in the replication of the 40-s delay. In discussing the presence or absence of elevated initial-link responding across conditions, I will use the results of the sign test.

Figure 5 shows the difference scores in graphic form. The data from the replication of the 40-s condition are indicated by empty symbols. For the purposes of fitting the line function to the data, the mean of the median difference scores from the two 40-s conditions was used. For all rats, initial-link responding was elevated at the 0 -s delay and the degree of elevation was quite large. For Rats $\mathrm{C} 1$ and $\mathrm{C} 4$, initial-link responding was elevated in all conditions, but this was not the case for Rat C7 and C10. Oddly, Rat C10 showed elevated initial-link responding in the original exposure to the 40-s condition, but not in the replication of this condition. Perhaps high response rates engendered by the 0 -s condition (which was presented second to this rat) increased 


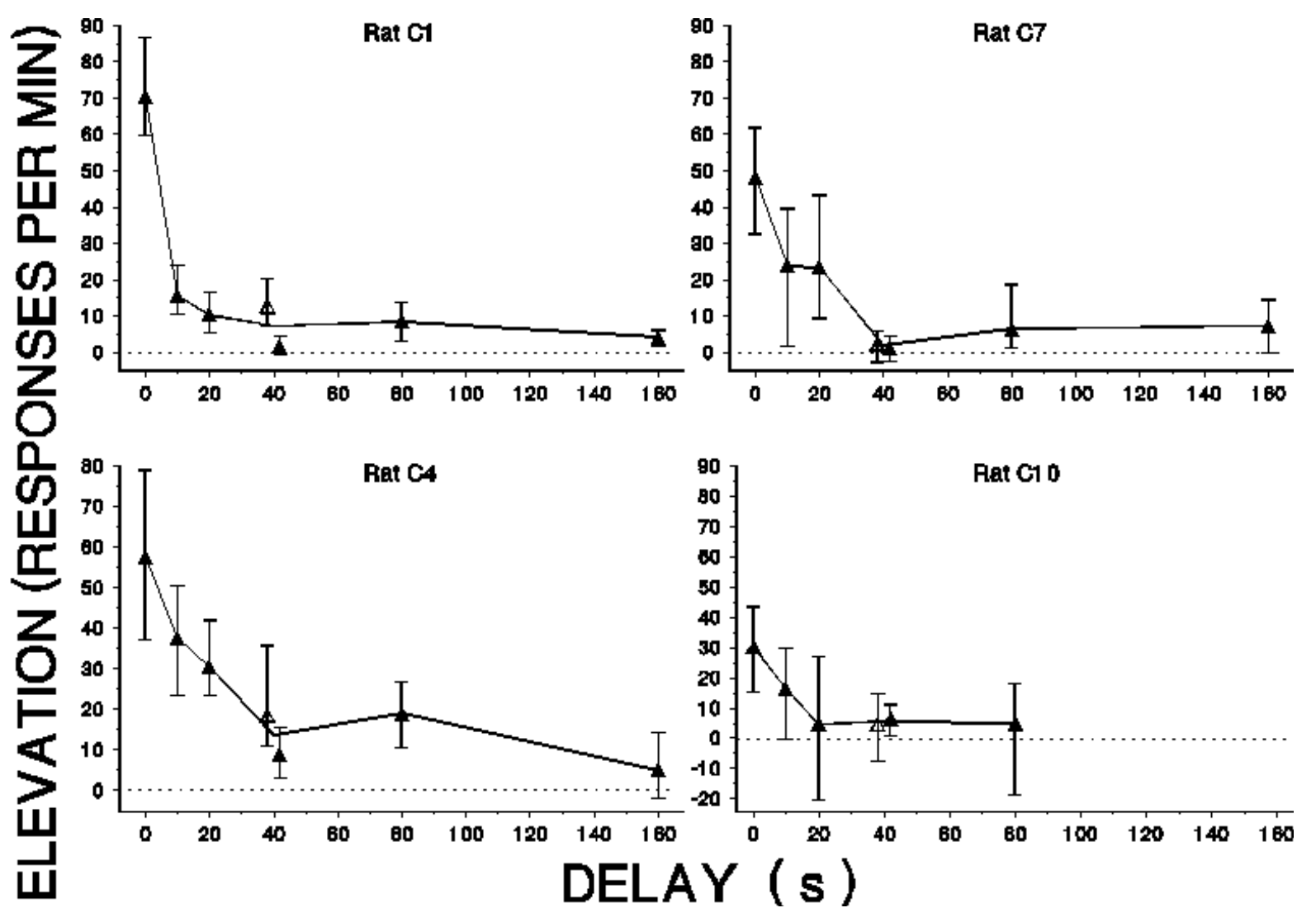

Figure 5. Experiment 2. Median response rate difference score (initial-link responses per min minus baseline responses per min) for each rat in each delay condition. The interquartile range of difference scores is indicated by the error bars. The dotted horizontal line at zero is provided for reference and represents no difference between initial-link and baseline response rate. The line function connects the median difference score from each delay condition, except at $40 \mathrm{~s}$, in which the mean of both median difference scores was used. 
the variability in responding overall, which precluded the detection of enhanced initial-link responding in most subsequent conditions, including the replication. Baseline response rates (see Figure 3) never returned to the low levels observed in the first condition, supporting this view. The high rates of responding reinforced in the initial link under the 0-s delay appear to have generalized to other portions of the session, and this pattern remained across the subsequent conditions. Thus, the behavior of Rat C10 was least sensitive to the initial-link contingency. Rats $\mathrm{C} 1$ and C4 showed greater elevation of initial-link responding in the replication of the 40-s condition than in the first exposure to the 40-s condition. Thus, a history effect was discernible for these two rats. Rat C7 showed a small but reliable elevation in the original 40-s condition, but not in the replication of that condition. This is particularly puzzling given that reliable elevations were observed at delays of 80 and $160 \mathrm{~s}$, and these elevations at longer delays were slightly greater in magnitude than that observed at $40 \mathrm{~s}$. Overall, the degree to which initial-link responding was enhanced was a clear function of the terminal-link delay. The precise delays at which responding was elevated differed across rats. All rats showed reliably elevated responding at delays of 0 and $10 \mathrm{~s}$. Two rats $(\mathrm{C} 1$ and $\mathrm{C} 4)$ exhibited elevated responding across the entire range of delays studied, and for Rat C7 responding was elevated in all but the replication of the 40-s condition. In general, initial-link elevation followed a negatively decelerating function. Rats $\mathrm{C} 1, \mathrm{C} 7$, and $\mathrm{C} 10$ had steep decay functions and Rat C4 had a shallower decay function, suggesting that Rat C4 may have been most sensitive to the delayed consequence.

Because the latency to respond after the onset of the initial-link stimulus may index sensitivity to the initial-link contingency, the latency in each of the 72 initial-link periods in the last 6 sessions of each condition was analyzed. To provide a baseline from which to assess 
control of initial-link latencies, baseline latencies were also calculated. Although no stimulus change accompanied the beginning of the 1-min baseline period, the time between the start of the baseline period and the first response equaled the baseline latency. For each adjacent pair of baseline and initial-link periods, the initial-link latency was subtracted from the baseline latency to determine control by the initial-link contingency. Thus, the analysis of latencies used difference scores similar to those in Table 2 and Figure 5. In the present case, difference scores lower than 0 indicate shorter latencies in initial link than in baseline, suggesting control by the delayed consequence. Table 3 shows the median and interquartile range of the baseline and initial-link latencies and of the aforementioned difference scores (initial-link minus baseline latency). Figure 6 shows the median and interquartile range of difference scores in graphic form. For Rat C1, latencies were longer in initial link than in baseline in all conditions. This result is opposite of what one would predict given sensitivity to the delayed consequence. Rat C4 exhibited shorter latencies in initial link than in baseline in all conditions except the original 40-s condition, in which the two latencies were similar. For Rat C10, shorter initial-link latencies were observed only in the original 40-s condition. Rat C7 had shorter initial-link latencies in all but the 0 - and 20-s delay conditions. General statements regarding the effects of delay on initiallink latency are not possible given these mixed results between and within subjects. One conclusion that can be made is that the elevated response rates in the initial link were not due to differences in latency. Indeed, initial-link latencies (Table 3) were similar across the range of delays studied and were not consistently shorter than baseline latencies. The delay variable affected the rate at which responses were emitted during the initial link, but did not affect the latency to begin that sequence of initial-link responses. 
Table 3

Order of conditions and the median and interquartile ranges of baseline and initial-link latency for each condition, in s. Also shown are the median and interquartile range of difference scores (initial-link minus baseline latency), in s.

\begin{tabular}{|c|c|c|c|c|c|c|c|c|c|}
\hline \multirow{3}{*}{$\frac{\text { Rat }}{\text { C1 }}$} & \multirow{3}{*}{$\frac{\text { Delay }}{40}$} & \multicolumn{8}{|c|}{ Latency (s) } \\
\hline & & \multicolumn{2}{|r|}{ Baseline } & \multicolumn{2}{|c|}{ Initial-Link } & \multicolumn{4}{|c|}{ Difference Score } \\
\hline & & 4.90 & $(2.44-10.80)$ & 6.93 & $(3.67-15.43)$ & 2.48 & $(-4.35$ & to & $10.60)$ \\
\hline & 20 & 3.03 & $(1.52-5.63)$ & 5.31 & $(2.13-7.25)$ & 1.34 & $(-2.26$ & to & $4.89)$ \\
\hline & 10 & 3.17 & $(1.15-7.75)$ & 4.20 & $(2.00-6.93)$ & 0.67 & $(-2.40$ & to & $3.21)$ \\
\hline & 0 & 2.06 & $(1.04-4.88)$ & 3.56 & $(2.33-4.38)$ & 0.65 & $(-1.58$ & to & $2.30)$ \\
\hline & 80 & 3.64 & $(1.78-7.14)$ & 3.92 & $(2.38-5.59)$ & 0.13 & $(-3.68$ & to & $2.83)$ \\
\hline & 160 & 4.36 & $(1.53-7.09)$ & 4.22 & $(2.60-6.49)$ & 0.10 & $(-3.26$ & to & $3.25)$ \\
\hline & 40 & 4.53 & $(1.66-7.40)$ & 3.16 & $(1.49-7.07)$ & 0.06 & $(-4.05$ & to & $2.75)$ \\
\hline \multirow[t]{7}{*}{ C4 } & 40 & 3.41 & $(1.57-6.75)$ & 4.20 & $(2.03-7.53)$ & 0.06 & $(-4.72$ & to & $3.96)$ \\
\hline & 20 & 1.74 & $(0.67-4.06)$ & 1.62 & $(1.09-2.84)$ & -0.18 & $(-1.68$ & to & $1.20)$ \\
\hline & 10 & 1.83 & $(0.98-3.35)$ & 1.54 & $(1.18-1.95)$ & -0.46 & $(-1.67$ & to & $0.66)$ \\
\hline & 0 & 1.58 & $(0.68-3.17)$ & 1.75 & $(1.14-2.46)$ & -0.15 & $(-2.21$ & to & 1.07) \\
\hline & 80 & 2.63 & $(0.91-7.45)$ & 2.03 & $(1.43-3.26)$ & -0.71 & $(-5.45$ & to & 1.43) \\
\hline & 160 & 2.64 & $(0.52-4.94)$ & 1.63 & $(1.14-3.07)$ & -0.29 & $(-2.75$ & to & $1.29)$ \\
\hline & 40 & 2.41 & $(0.50-5.06)$ & 1.74 & $(1.31-2.40)$ & -0.62 & $(-3.01$ & to & $1.20)$ \\
\hline \multirow[t]{7}{*}{ C7 } & 40 & 3.51 & $(1.29-5.00)$ & 3.24 & $(1.22-5.74)$ & -0.16 & $(-2.93$ & to & $2.03)$ \\
\hline & 0 & 1.32 & $(0.29-3.11)$ & 2.24 & $(0.68-3.14)$ & 0.14 & $(-0.98$ & to & $2.26)$ \\
\hline & 10 & 0.97 & $(0.24-4.12)$ & 1.48 & $(0.54-3.65)$ & -0.10 & $(-2.60$ & to & $1.42)$ \\
\hline & 20 & 1.49 & $(0.50-3.15)$ & 3.11 & $(0.93-5.29)$ & 0.85 & $(-0.37$ & to & $2.92)$ \\
\hline & 80 & 1.92 & $(0.65-3.55)$ & 1.06 & $(0.57-2.09)$ & -0.31 & $(-2.69$ & to & $0.75)$ \\
\hline & 160 & 2.74 & $(1.18-4.96)$ & 1.68 & $(0.84-4.08)$ & -0.51 & $(-2.91$ & to & $1.57)$ \\
\hline & 40 & 3.00 & $(1.47-5.03)$ & 1.90 & $(0.85-3.53)$ & -0.32 & $(-3.46$ & to & $0.94)$ \\
\hline \multirow[t]{6}{*}{ C10 } & 40 & 3.17 & $(1.24-6.55)$ & 3.90 & $(1.25-8.43)$ & -0.36 & $(-3.71$ & to & $2.64)$ \\
\hline & 0 & 1.42 & $(0.40-3.71)$ & 4.11 & $(2.52-5.68)$ & 1.93 & $(-0.71$ & to & $4.44)$ \\
\hline & 10 & 2.16 & $(0.43-5.42)$ & 3.08 & $(0.91-6.28)$ & 0.06 & $(-2.58$ & to & $4.24)$ \\
\hline & 20 & 2.04 & $(0.35-4.82)$ & 3.90 & $(1.25-8.43)$ & 1.34 & $(-1 \cdot 84$ & to & $5.93)$ \\
\hline & 80 & 2.22 & $(0.51-6.99)$ & 1.74 & $(0.81-4.50)$ & 0.17 & $(-5.47$ & to & $2.16)$ \\
\hline & 40 & 2.53 & $(0.53-10.42)$ & 4.77 & $(1.94-12.45)$ & 1.47 & $(-5.56$ & to & $8.64)$ \\
\hline
\end{tabular}




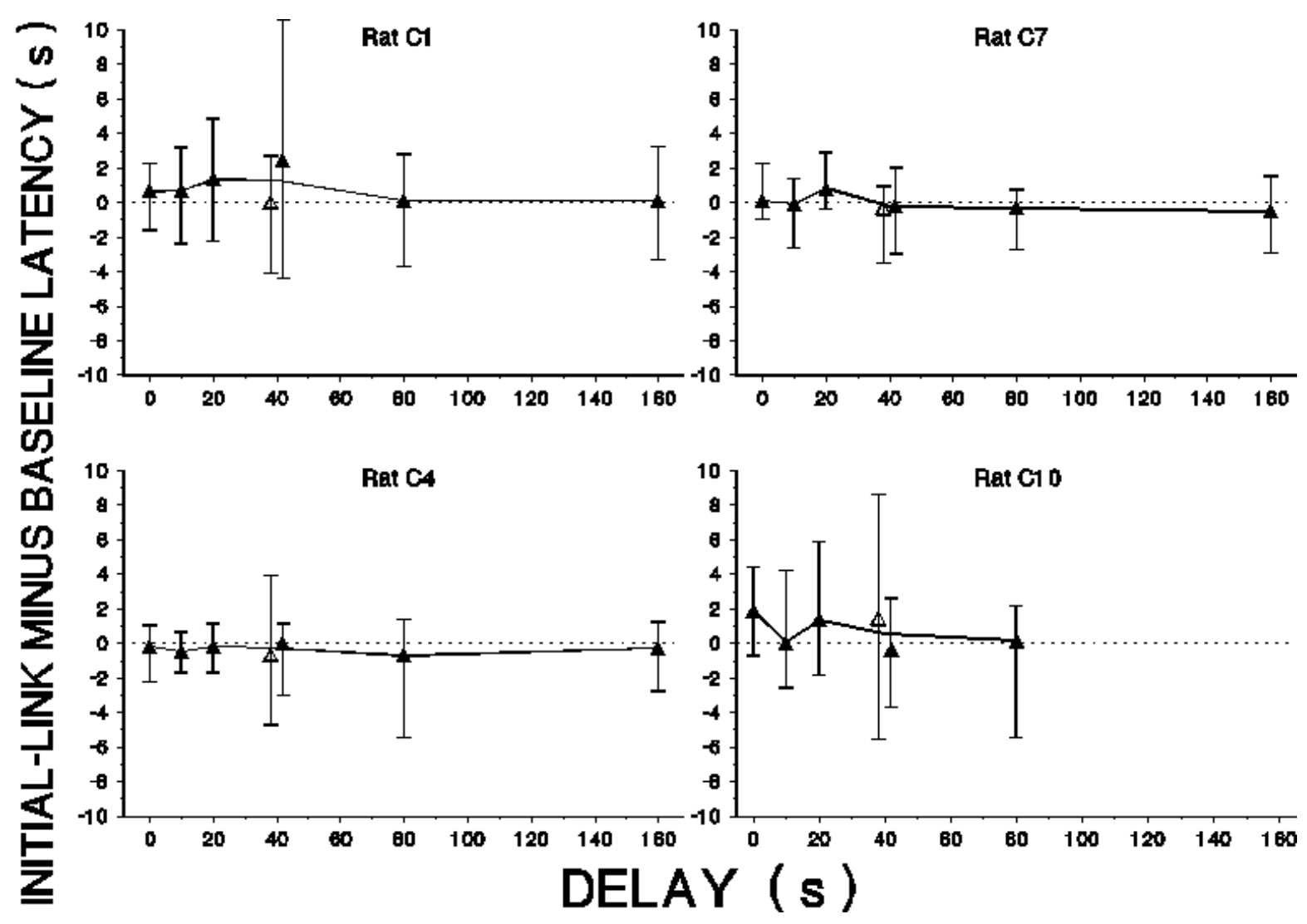

Figure 6. Experiment 2. Median latency difference score (initial-link latency minus baseline latency, in s) for each rat in each delay condition. The interquartile range of difference scores is indicated by the error bars. The dotted horizontal line at zero is provided for reference and represents no difference between initial-link and baseline latency. The line function connects the median difference score from each delay condition, except at $40 \mathrm{~s}$, in which the mean of both median difference scores was used. 


\section{Discussion}

The present results revealed control of aggregated responses by a delayed food reinforcer. Initial-link response rates and the degree of initial-link elevation (in responses per min) were highest at the shortest (0-s) delay for all rats. A small degree of elevation was detectable at long delays (80 and $160 \mathrm{~s}$ ) for three rats, but was absent at a delay of $80 \mathrm{~s}$ for Rat C10. For two rats, elevated initial-link responding was not relibale at a delay of $40 \mathrm{~s}$ given the absence of elevated initial-link responding in the replication of that condition. The latency data revealed no consistent effect of the delay to reinforcement. For only one rat (C4), latencies were generally shorter in initial link than in baseline. Overall, the results suggest a finite temporal window over which aggregated responses can be controlled by a delayed reinforcing consequence. For two rats, however, the temporal limits were not reached in the present study. The fact that initial-link elevation was large at short delays and small or absent at long delays provides evidence for the assertion that aggregated responses can be controlled by a delayed consequence, but consequences delayed on the order of minutes or more are likely to exert only weak control over such responses.

These results are consistent with those from other paradigms designed to assess control by delayed reinforcers. For example, studies of acquisition with delayed reinforcement, although typically examining control of a single response rather than an aggregation of responses, are similar to the present experiment. Both procedures arrange a delayed consequence contingent upon responding. The discrete intial-link stimulus and background reinforcement schedule used in the present procedure are significant departures from these acquisition studies, which could be viewed as arranging a background schedule of extinction. Further, many such acquisition studies 
arrange resetting delays to reinforcement that were not used in this experiment, but both procedures control the temporal separation between effective responses and reinforcement. Despite the differences, the findings were generally similar across both paradigms. Delays as long as $30 \mathrm{~s}$ lead to acquisition of responding in rats (Critchfield \& Lattal, 1993; Lattal \& Gleeson, 1990; Lesage et al., 1996; Wilkenfield et al., 1992). In the present experiment, acquisition of elevated response rates in the initial link was observed at a delay of $40 \mathrm{~s}$ (the first experimental condition) for all rats. The control observed at delays of 80 and $160 \mathrm{~s}$ for three rats may have resulted from experience with short delays, which may have overestimated sensitivity to the delayed reinforcer. Although such history effects must be acknowledged, the data from these rats suggests control at delays as long as $160 \mathrm{~s}$, which are quite long given the aforementioned findings from acquisition studies using delayed reinforcement.

The use of a large reinforcer may be responsible for the enhanced control observed. Not only should the delivery of six pellets function as a stronger reinforcer than the delivery of one pellet, this stimulus event should be more salient than the delivery of fewer pellets. This is particularly true given a background of immediate, 1-pellet reinforcers. It would be interesting to examine the effects of manipulating the magnitude of the delayed reinforcer. In a similar vein, the effects of various background rates of reinforcement could be studied by manipulating the baseline VI schedule. Indeed, this latter manipulation could provide a more sensitive assay of control by delayed reinforcement. The present experiment arranged a VI 150-s schedule to maintain steady and moderate response rates. This was designed to ensure completion of the initial-link ratio within a minute or less. A leaner baseline schedule would be expected to maintain lower rates of responding against which elevated initial-link responding might be 
detected more easily. However, very lean schedules may lead to long periods with little or no responding and may increase variability in response rates, compromising the detection of reliable response rate differences between baseline and initial link.

Overall reinforcement rate (total pellets divided by session time) varied across the delay conditions. This is because short programmed delays to the large reinforcer reduced terminal-link duration, thereby decreasing the overall length of the session. Furthermore, the shorter terminallink delays to reinforcement maintained higher response rates in the initial-link, reducing initiallink duration and, thus, session duration. Reinforcement rates ranged from about 1.0 to 1.3 reinforcers per min across conditions. At the shorter delays, the higher absolute reinforcement rate may have led to higher response rates overall, particularly given that the relative increase in reinforcement rate was sizable (i.e., 30\%). However, this interpretation seems unlikely given that terminal-link response rates decreased at the shorter delays for most rats. Thus, a generalized increase in responding with increased initial-link response rate does not explain the increased baseline responding and the decreased terminal-link responding. Also, the delay variable affected initial-link response rates more than any other, suggesting that changes in delay and not overall reinforcement rate, were responsible for changes in response rates. Because reinforcement rate and the interval between initial-link presentations were confounded, it is impossible to determine which variable was responsible, if either was. Future studies using this procedure should reduce or eliminate the confound between delay to reinforcement and overall reinforcement rate. One method for equating reinforcement rate across different delays would be to fix the duration of the terminal link and vary the temporal placement of the delayed reinforcer. This would reduce interpretative difficulties that may stem from variations in reinforcement rate across different 
delay conditions.

Perhaps a more satisfactory explanation of high baseline response rates at short delays could be based on discrimination. That baseline response rates increased with initial-link response rates as delay decreased suggests that high response rates engendered by the initial-link contingency generalized to other periods, including baseline. This view is only partly supported because terminal-link response rates were lower than baseline rates at short delays for most rats. In fact, terminal-link response rates generally increased with increased delays, a pattern opposite that of baseline and initial-link rates. Terminal-link response rates were usually lower than baseline and initial-link rates at short delays, demonstrating that the rats discriminated the terminal-link from other periods at short delays. The present procedure might be improved by increasing the disparity between the baseline and initial-link stimuli or by increasing the salience of one or both stimuli. For example, signaling the initial link with an audible tone might improve the discrimination of the various components by providing a qualitatively different stimulus from the visual houselight stimulus used to signal the baseline and terminal-link periods. Indeed, some evidence suggests that auditory stimuli are more discriminable to albino rats (Field \& Boren, 1963; Osborne \& Shelby, 1975). Further dissociating baseline from initial link should decrease correlated changes in response rates and improve detection of control by the delayed consequence.

One variation of the present procedure that might shed light on the possibility of generalized response rates would be to change the response requirement in the initial link. By making access to the terminal link dependent upon pausing (i.e, a differential-reinforcement-ofother-behavior, DRO, schedule), sensitivity to the initial-link contingency could be measured by 
a relative decrease in response rate from baseline to initial link. Greater suppression (i.e., relatively lower initial-link response rates) would be expected as delay decreased. Generalized suppression of baseline responding would be evidenced by lower baseline response rates in DRO conditions than in corresponding FR conditions programming identical delays. An advantage of this variation is that the pausing engendered by the DRO requirement is incompatible with earning baseline reinforcers. The contingency would require the subject to choose between fulfilling the initial-link requirement by pausing and responding on the baseline schedule of immediate reinforcement. If initial-link response rates were lower than baseline rates despite these competing contingencies, control by the delayed consequence would be even more convincing. The DRO procedure might also overcome a problem with the present procedure. A general acceleration of responding with the passage of time would produce higher initial-link response rates, and under the FR contingency, one might conclude control by the delayed consequence even if responding was not under such control. In contrast, this same pattern of responding under the DRO contingency would reveal clear insensitivity to the delayed consequence. A further possibility would be to alternate the FR and DRO requirements while maintaining a particular terminal-link delay. This reversal design would allow one to demonstrate elevation and suppression of initial-link responding at the same delay value. Such a reversal design could provide more convincing evidence of sensitivity to changes in the initial-link contingency under a particular delay to reinforcement.

Unfortunately, the interpretation of the present results is somewhat ambiguous with respect to the limits of control by temporally extended response-reinforcer relations. One interpretation is that extraordinary sensitivity to such relations was observed compared to other 
assays of control by delayed reinforcement. Another interpretation is that elevated initial-link responding was established at short delays, and this pattern perseverated as longer delays to reinforcement were introduced. The statistical analysis does not settle the issue and only demonstrates that elevations were present at long delays for most rats. Demonstrations of experimental control are necessary. One such manipulation, discussed above, is the reversal of the response requirement in the initial link from FR to DRO and back again. Another control condition would be to reverse the stimuli differentiating initial link from other periods. Another approach might be to eliminate the large reinforcer while maintaining the same stimuli and response-produced stimulus changes. This "sham reinforcement condition" is less elegant, however, because the overall reinforcement rate would be much lower due to omission of the large reinforcers, which comprised a majority of all pellets earned. Regardless of which approach is taken, the present experiment requires a suitable control or comparison condition to assess whether sensitivity is reliable at long delays to reinforcement.

The order of conditions may affect assessments of sensitivity to the delayed consequence. In the present study, elevated initial-link responding was established early in training. As longer delays to reinforcement were arranged the degree of elevation diminished. However, long delays capable of maintaining elevated responding may not be sufficient to produce elevated responding. The present procedure may have overestimated sensitivity to delayed reinforcement by presenting short delays before longer delays. Furthermore, longer delays were presented in ascending order and this may have increased sensitivity at long delays. However, the changes in delay were large and sudden rather than small and gradual, so the extent to which order of conditions may have enhanced sensitivity to long delays seems minimal. Indeed, only half of the 
subjects showed greater initial-link elevation during the replication of the 40-s condition than during the original exposure. The other two rats exhibited elevated responding in the original 40s condition, but not during the replication. One of these rats (C7) had reliable elevations at delays of 80 and $160 \mathrm{~s}$. This suggests that elevated initial-link responding diminished with time, and the order of conditions may have overestimated control. Two modifications to address this possibility would be to run conditions for more sessions and to present delays in descending order.

Another interpretation of the control at long delays rests on conditioned reinforcement. The present procedure was designed to preclude interpretations of performance based on immediate consequences of responding by using an unsignaled delay to reinforcement in the terminal link. Recall that the houselight accompanied all periods of the session except the initial link. Providing a unique stimulus correlated with the terminal link would have likely established that stimulus as a conditioned reinforcer through classical conditioning (due to a reliable and contiguous relation to food). Then, initial-link responses could be reinforced immediately by presentation of terminal-link stimulus. Although no unique stimulus was correlated with the terminal link in the present experiment, the offset of the initial-link reliably preceded delivery of the large reinforcer (the putative unconditional stimulus, US). At short delays, the offset of the initial-link stimulus was also contiguous with the delivery of the large reinforcer. This is analogous to a delay-conditioning procedure, the most effective Pavlovian procedure, in which little time separates conditional stimulus (CS) offset and US onset. This relation should establish offset of the initial-link stimulus as a conditioned reinforcer. At longer terminal-link delays, the procedure is analogous to a trace-conditioning procedure, in which a sizable delay (the trace 
interval) separates CS offset from US onset. Trace conditioning is generally less effective than delay conditioning and the degree of conditioning decreases as the trace interval increases. Although the limits of trace conditioning are not established, the autoshaping of pigeons has been established with trace intervals of 28 s (Newlin \& Lolordo, 1976) and 36 s (Lucas, Deich, \& Wasserman, 1981) and maintained with trace intervals as long as $120 \mathrm{~s}$ (Lucas et al., 1981). Thus, even rather long trace intervals may have been effective in establishing and maintaining a conditioned reinforcing function of initial-link stimulus offset.

The above interpretation fits the data well. The degree of response elevation in the initial link was greatest at the shortest delays and smallest at the longest delays. The conditioned reinforcing function of initial-link stimulus offset should decrease as the delay between that stimulus change and the presentation of large reinforcer increases. Thus, the immediate conditioned reinforcement provided by entry into the terminal link would be a direct function of the terminal-link delay, producing a high degree of elevation at short delays and little or no elevation at long delays. This was the pattern of results in Experiment 2. Similar results have been obtained under VI schedules ending in briefly signaled (Schaal \& Branch, 1988, 1990) or unsignaled (Williams, 1976; Sizemore \& Lattal, 1978) non-resetting delays to food. These studies have shown that response rates generally decrease as programmed response-reinforcer or CS-US delays increase.

With appropriate modifications, the present procedure could address the possible role of conditioned reinforcement in controlling initial-link responding. To test control of initial-link responding by initial-link stimulus offset, that stimulus change could be withheld until after delivery of the large reinforcer. This should eliminate any response-dependent conditioned 
reinforcement for initial-link responding while maintaining the relation between initial-link responding and delivery of the large reinforcer. If the degree of elevation in initial link is reduced relative to conditions that provide the stimulus change contingently, the results would be consistent with a conditioned reinforcement view. In the absence of the stimulus change, the relation between initial-link responding and the large reinforcer could be manipulated. For example, the large reinforcer could be delivered response-independently, perhaps by yoking the obtained times to complete the initial link from a preceding response-dependent condition. Comparing response-dependent and response-independent conditions should reveal whether initial-link responding is elevated in the absence of conditioned reinforcement and only when such responding actually produces the large, delayed reinforcer.

The present experiment should be extended to address interpretations of the results based on conditioned reinforcement. Without appropriate comparison conditions, the present results could be interpreted as demonstrating control by temporally extended response-reinforcer relations, temporally extended CS-US relations, or both.

\section{Experiment 3}

In Experiment 3, control of choice by delayed reinforcement was investigated using an adjusting-delay procedure based on the one developed by Mazur (1987). On each trial, a choice between a small and a large reinforcer was presented. The delay between a choice response and reinforcement was equal for both alternatives but was adjusted across trials based on choice behavior. The magnitude of the small reinforcer was held constant at one pellet and the magnitude of the large reinforcer was to be manipulated across conditions from two to six pellets. The indifference point, the delay at which the small and large reinforcer are chosen about 
equally often, was to provide an index of the delay at which behavior is no longer affected by differential reinforcement. Unlike Experiment 1, the procedure arranged forced trials that ensured contact with the delayed consequence of each response. Unlike Experiment 2, a single response rather than several responses was sufficient to produce the delayed consequence. Moreover, due to the titrating aspect of the procedure, the delay at which behavior is no longer sensitive to its consequences can be pinpointed more precisely than in Experiments 1 and 2. Unfortunately, Experiment 3 failed to produce reliable preference for the large reinforcer, even at short delays, preventing the determination of delays at which differential reinforcement no longer functions as such.

Method

$\underline{\text { Subjects and Apparatus }}$

Five experimentally naive male Sprague-Dawley rats were used. The rats were about 90 days old at the start of the experiment and were maintained at approximately $80 \%$ of free-feeding weight via daily post-session feedings given at least 30 min after being returned to their home cages. The apparatus was the same as in Experiment 2.

\section{$\underline{\text { Procedure }}$}

Preliminary training. Each rat was taught to approach the food tray and promptly consume the pellet upon delivery. Lever pressing was established by reinforcing successive approximations to this behavior. The position of the lever to which initial shaping occurred was counterbalanced across rats. After 100 reinforced lever presses the session terminated. The subsequent session was used to shape responding to the other lever and terminated after 100 reinforced presses. During shaping, only the cue light above the effective lever provided 
illumination and only the effective lever was present.

Several variations of procedure were studied in an attempt to establish preference for the large reinforcer. The order of conditions and the number of sessions in each are shown in Table 4. Each experimental condition (procedural variation) is described below.

Adjusting-delay procedure. Each session consisted of several discrete trials. Both levers were present. The cue light above a particular lever was correlated with the reinforcer magnitude provided by that lever. For some rats, a flashing cue light signaled the lever providing the larger reinforcer magnitude and a constant cue light signaled the lever providing the smaller reinforcer magnitude. For the other rats, the stimulus-magnitude relation was opposite. The position of the flashing and constant cue light varied pseudorandomly across trials.

The first two trials of each session were forced trials. Only one of the two response alternatives produced reinforcement. The effective alternative was signaled by the appropriate cue light (flashing or constant) above the effective lever (left or right). Which alternative was presented first and the position in which it was presented were programmed pseudorandomly. For each pair of forced trials, each stimulus was presented once and the position differed for the two alternatives. On the next pair of forced trials a different position/order combination was presented. Thus, the four types of forced trial pairs were presented equally often (e.g., flashing presented first on the left) for every eight pairs of forced trials completed. Neither stimulus was presented on the same side more than twice in succession.

During a forced trial, a response on the effective lever turned off the corresponding cue light and initiated a signaled delay to reinforcement during which the houselight was on. The signal during the delay was identical for the small and large reinforcer outcomes. At the end of 
Table 4

Experiment 3. The order of conditions and number of sessions in each for each rat.

\begin{tabular}{lccccc}
\hline & \multicolumn{5}{c}{ Rat } \\
\cline { 3 - 6 } \multicolumn{1}{c}{ Condition } & $\underline{\mathrm{A} 2}$ & $\underline{\mathrm{A} 3}$ & $\underline{\mathrm{A} 5}$ & $\underline{\mathrm{A} 8}$ & $\underline{\mathrm{A} 9}$ \\
\cline { 1 - 3 } Adjusting Delay (AD) & 2 & -- & 3 & -- & 3 \\
Forced Only & 5 & 5 & 5 & 5 & 5 \\
Adjusting Delay (AD) & 6 & 4 & 6 & 5 & 6 \\
Fixed Delay 1 s (FD1) & 10 & 9 & 10 & 9 & 10 \\
Fixed Delay 0 s (FD0) & 5 & 5 & 5 & 5 & 5 \\
90-s ITI (90) & 2 & 2 & 2 & 2 & 2 \\
Go/No-go & 17 & 17 & 17 & 17 & 17 \\
& & & & & \\
\hline
\end{tabular}


the delay, the reinforcer cycle began. For the small reinforcer, one pellet was delivered and the houselight was turned off. For the large reinforcer, pellets were delivered at 1-s intervals and the houselight was turned off when the last pellet was delivered. A 45-s intertrial interval (ITI) followed each reinforcement cycle. During the ITI the houselight remained off. A response to either lever during the ITI reset the ITI. The onset of one or more cue lights marked the end of the ITI and the beginning of the next trial (either a forced trial or a choice trial).

On choice trials, both cue lights were activated and a response to either lever resulted in reinforcement. The first response terminated both cue lights and initiated the signaled delay to reinforcement. After the delay, the appropriate reinforcement cycle was presented, followed by the ITI. The position of the flashing and constant cue lights varied pseudorandomly across choice trials. The same stimulus was presented no more than thrice in the same position across the last six choice trials.

Choice trials were presented in pairs. If the rat chose the same reinforcer during the two choice trials, an intervening pair of forced trials followed. If the rat chose the large reinforcer on both choice trials, the delay to both reinforcers increased by $2 \mathrm{~s}$. If the rat chose the small reinforcer on both choice trials, the delay to reinforcement decreased by $2 \mathrm{~s}$ to a minimum value of $1 \mathrm{~s}$. In either case, the new delay was presented in the two forced trials that followed. If the rat did not prefer one alternative over the other (each reinforcer was chosen once in the pair of choice trials), the delay to reinforcement was unchanged and another pair of choice trials followed with no intervening forced trials. The forced trials ensured that the rat contacted both alternatives before each pair of choice trials.

The first pair of choice trials completed after delivery of 140 pellets ended the session. In 
the subsequent session, the delay to reinforcement for the first four trials (two forced and two choice trials) equaled the terminal delay of the preceding session.

The magnitude of the large reinforcer was to be manipulated across conditions while the magnitude of the small reinforcer was always one pellet. Each rat was assigned randomly to a different magnitude condition (Rat A2=2; Rat A8=3; Rat A5=4; Rat A3=5; Rat A9=6) at the outset of experimentation. Due to difficulties in establishing discriminated performance, the within-subjects examination of various magnitudes was abandoned eventually, and all subjects were studied with the 5- or 6-pellet reinforcer to make the consequences of each choice more disparate and facilitate the acquisition of a preference for the large reinforcer.

Because preference for the large reinforcer was not established, several procedural variations were studied. Each was designed to mitigate position biases and establish discriminative control by the cue lights, thereby facilitating the development of a preference for the large reinforcer.

Forced-only procedure. The aforementioned adjusting-delay procedure was modified such that only forced trials were presented. The magnitudes of the small and large reinforcers for each rat were the same as above and the delay to reinforcement was held constant at $1 \mathrm{~s}$ on all trials. By requiring frequent switching between levers and by maintaining a brief delay to reinforcement this forced-only procedure was designed to reduce position biases and bring responding under discriminative control of the cue lights. By examining the number of responses on an inactive lever (during the ITI and during forced trials) and the latency to respond on all trials, improvement at the task could be measured. This procedure remained in effect for 5 sessions, until few responses occurred on an inactive lever and latencies on most trials were $4 \mathrm{~s}$ or less. 
Fixed-delay procedure. The fixed-delay procedure was identical to the adjusting-delay procedure except that the delay to both reinforcers was constant across trials, regardless of choice performance. Unlike the forced-only procedure, this procedure allowed choice behavior to be examined and any preference for the large reinforcer could be detected. Because short delays should promote the acquisition of a discrimination between the small and large reinforcers, the delay was fixed at $1 \mathrm{~s}$. This constituted the FD1 condition. After several sessions with no obvious improvement, the fixed delay was reduced to $0.01 \mathrm{~s}$ in the subsequent fixed-delay condition (labeled FD0).

Intertrial interval manipulation. The fixed-delay of $0.01 \mathrm{~s}$ was maintained as in condition FD0, but the ITI was increased to $90 \mathrm{~s}$. Considerable evidence suggests that longer ITIs enhance control by stimulus-outcome relations (Mackintosh, 1983, 1974; Gormezano, Kehoe, \& Marshal, 1983; Gormezano \& Moore, 1969). Further, by reducing the rate of trials the obtained rate of reinforcement would be reduced. Under conditions in which the overall reinforcement rate is lower, local changes in rate may be more salient and discrimination of differential magnitude more likely. The ITI manipulation was unsuccessful, as were all of the aforementioned procedures, in producing a preference for the large reinforcer. For this reason, a single-lever procedure was implemented next to address the same question but reduce position bias as a potential hindrance to establishing preference for the large reinforcer.

Go/no-go procedure. Only the unpreferred lever was available (the other lever was removed). All trials were choice trials, in that behavior determined whether a small or large reinforcer was delivered. Each choice trial consisted of a choice period that lasted exactly $4 \mathrm{~s}$ and then the appropriate reinforcement cycle (small or large), followed by a 60-s ITI. The start of the 
choice period was signaled by the onset of the cue light above the lever. For the flashing group, a flashing cue light signaled that one or more responses during the choice period would produce the large reinforcer. Conversely, one or more responses in the presence of a constant cue light produced a small reinforcer at the end of the choice period. Failing to emit one response during the choice period produced the other reinforcer at the end of the choice period. In this way, a rat could choose the large reinforcer on every trial by responding promptly when the flashing cue light was on and by not responding when the constant cue light was on. For the rats in the constant group, the stimulus-reinforcer relations were reversed (responding to a constant cue light produced a large reinforcer). Bias can also be assessed in the go/no-go procedure.

Responding versus not responding in this procedure is comparable to left versus right choices in the two-lever procedure. Thus, the proportion of active choices (rather than the proportion of left choices) was examined in the go/no-go procedure to index bias. To facilitate preference for the large reinforcer, the magnitude of the large reinforcer was set to 5 pellets (Rats A3, A5, and A9) or 6 pellets (Rats A2 and A8). The first pair of trials completed after 120 pellets had been delivered terminated the session.

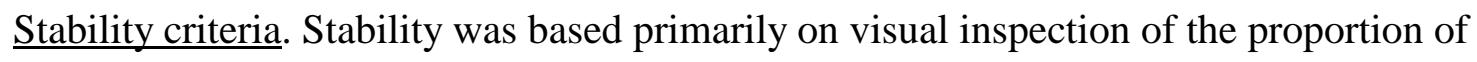
large reinforcers chosen in each session. Also, visual trends in the proportion of left or active choices had to be absent.

\section{Results}

Table 4 shows the order of conditions and the number of sessions in each for each rat. The three rats for which the large reinforcer was signaled by a flashing cue light (Rats A2, A5, and A9) were exposed to the adjusting-delay procedure for two to three sessions. Thereafter, all 
rats were treated similarly. The forced-only procedure was in effect for 5 sessions followed by a return to the adjusting-delay procedure. For the flashing group, this constituted a re-exposure to the adjusting-delay procedure (labeled AD2 in Figure 7) and for the constant group this was the first exposure. The condition lasted 4 to 6 sessions. The fixed-delay conditions were presented next, first with a constant delay of $1 \mathrm{~s}$ (FD1) and next with a delay of $0.01 \mathrm{~s}$ (FD0). Finally, the ITI was lengthened to $90 \mathrm{~s}$ for 2 sessions before the procedure was abandoned and the go/no-go procedure was studied. This final procedure remained in effect for 17 sessions.

Figure 7 shows the overall choice proportions in each session across all conditions, except the forced-only condition in which there were no choice trials, and therefore, no measures of choice. The proportion of left lever choices (or active choices in the go/no-go procedure) is represented by empty circles and the proportion of large reinforcer choices is represented by solid squares. For Rats A2, A5, and A9 the first two conditions in Figure 7 used the adjusting-delay procedure. The two conditions were separated by the forced-only procedure (from which the data are not shown). For Rats A3 and A8, only the first condition shown used the adjusting-delay procedure and this followed the forced-only procedure.

The proportion of left choices differed across rats. Rat A2 generally favored the left lever and Rat A9 favored the right. For the other three rats, preference for one lever over the other fluctuated broadly across sessions. For all rats, the proportion of choices for the large reinforcer approximated indifference at a value of .5. For Rats A3 and A8, a small but consistent preference for the large reinforcer was observed. Unfortunately, the degree of preference was small, never exceeding .75. In contrast, Rats A2 and A9 preferred the small reinforcer in a majority of sessions under the adjusting-delay procedure. For Rats A2 and A9 the adjusted delay remained 


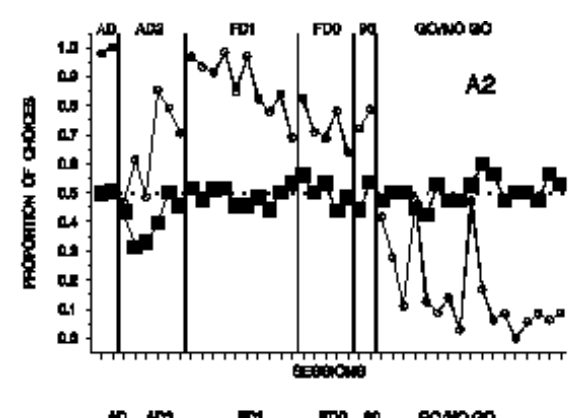

\section{$\multimap-$ LARGE ○ LEFT / ACTIVE}
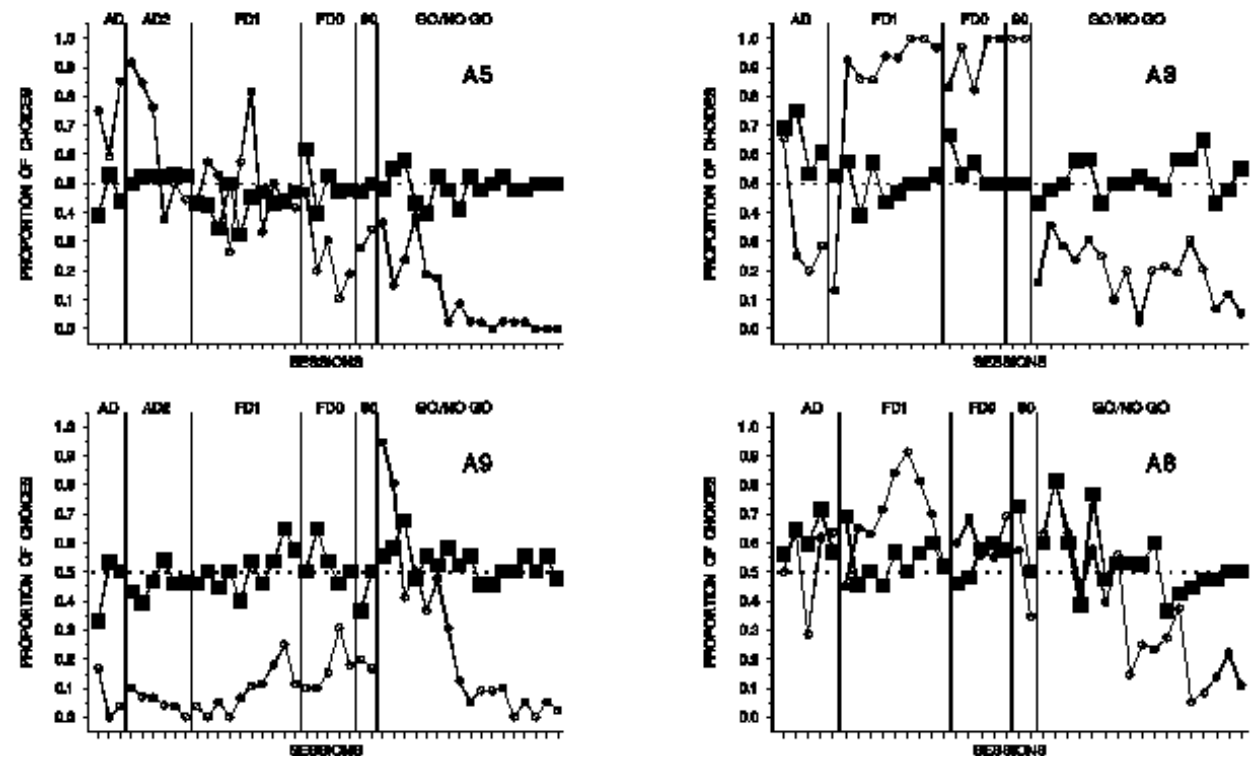

Figure 7. Experiment 3. Proportion of large reinforcer choices (squares) and of left lever choices (circles) are shown for each rat in each session. In the final condition, the Go/No-go procedure, circles represent the proportion of active choices. The dotted horizontal line at .5 indicates indifference (i.e., equal choice proportions). 
near the nominal value of $1 \mathrm{~s}$. For the other rats, the small degree of preference for the large reinforcer was sufficient to increase the delay within sessions. For the last session of the adjusting-delay condition, the final delays were 31,13 , and $51 \mathrm{~s}$ for Rats $\mathrm{A} 3, \mathrm{~A} 5$, and $\mathrm{A} 8$, respectively. This limited success was tempered by several other aspects of performance. For all rats, several responses occurred on inactive levers during the ITI and forced trials, suggesting poor discriminative control by the cue lights. Indeed, responses during the ITI postponed the onset of the next trial, but were frequent nevertheless. Responses on the inactive (unlit) lever on forced trials resulted in long latencies to press the active lever, postponing reinforcement and the next trial. Even for the three rats that preferred the large reinforcer enough to drive the delay upward, responses during the delay to reinforcement were numerous. This too suggested poor discriminative control by the cue-light stimuli. These observations, combined with position biases and little or no preference for the large reinforcer, made it clear that further training was necessary. The subsequent procedural variation was designed to establish discriminative control by the cue lights by maintaining a brief delay to reinforcement, which should in turn facilitate the development of a preference for the large reinforcer.

The fixed-delay condition removed the adjusting aspect of the schedule and maintained an equal delay to reinforcement of $1 \mathrm{~s}$ on forced and choice trials, regardless of preference. This was designed to establish reliably high preference for the large reinforcer without greatly modifying the procedure. When choice of the large reinforcer was consistently greater than .8 , the condition was to be terminated. This criterion was never met. The fixed delay was subsequently reduced to $0.01 \mathrm{~s}$ for several sessions. This too was unsuccessful in establishing preference for the large reinforcer. Next, the ITI was increased to $90 \mathrm{~s}$ for 2 sessions. Across 
these three conditions, choice proportions were unchanged, as shown in Figure 7. Choice of the large reinforcer approximated indifference and left choice proportions generally persisted. Thus, these procedural variations neither reduced position biases nor increased preference for the large reinforcer. Because position biases might hinder reliable preference for the large reinforcer, a go/no-go procedure was implemented to address the same research question using a one-lever choice paradigm.

No overall preference for the large reinforcer was observed in the two-lever choice procedures, but position biases were observed. A molecular analysis was performed to determine whether the small and large reinforcers were controlling choice within the session in a way that might not be revealed by overall choice measures. The position of the choice (left or right) was studied as a function of the position and magnitude (e.g., left-large) of the most recent reinforcer (i.e., the reinforcer from the preceding forced or choice trial). Thus, the probabilities of left and right choices were examined for each of four position-magnitude combinations: left-large, leftsmall, right-large, right-small. The results were aggregated across the last two sessions under the fixed-delay procedure (fixed-delay $=0.01 \mathrm{~s}$ and ITI $=90 \mathrm{~s}$ ). The tendency to perseverate on the lever that recently produced a large reinforcer was indexed by summing the probabilities of a left choice given a large reinforcer on the left (i.e., left|left-large) and of a right choice given a large reinforcer on the right (right|right-large). The tendency to perseverate was also calculated for small reinforcers in a similar way (left|left-small plus right|right-small). The tendency to switch from the lever that recently delivered a large reinforcer (left|right-large plus right|left-large) and the tendency to switch from the lever that recently delivered a small reinforcer (left|right-small plus right|left-small) were also calculated. Table 5 shows the individual and summed choice 
Table 5

Experiment 3. Probability of left (L) or right (R) choice following a particular position-magnitude combination (LL, left-large; LS, left-small; RL, right-large; RS, right-small). Summed probabilities across the left and right levers are listed under Perseverate and Switch for both reinforcer types, Small and Large. Perseverate Small equals L|LS plus R|RS. Perseverate Large equals L|LL plus R|RL. Switch Small equals L|RS plus R|LS. Switch Large equals L|RL plus R|LL. See text for details.

Probability

Perseverate Switch

$\underline{\text { Rat }} \underline{\mathrm{L} \mid L L} \underline{\mathrm{L} \mid \mathrm{LS}} \underline{\mathrm{L} \mid \mathrm{RL}} \underline{\mathrm{L} \mid \mathrm{RS}} \underline{\mathrm{R} \mid \mathrm{LL}} \underline{\mathrm{R} \mid \mathrm{LS}} \underline{\mathrm{R} / \mathrm{RL}} \underline{\mathrm{R} / \mathrm{RS}} \quad \underline{\text { Small }} \underline{\text { Large }} \underline{\text { Small }} \underline{\text { Large }}$

$\begin{array}{lllllllllllll}\text { A2 } & .23 & .27 & .12 & .12 & .11 & .06 & .03 & .04 & .31 & .26 & .18 & .23 \\ \text { A3 } & .44 & .44 & .06 & .06 & .00 & .00 & .00 & .00 & .44 & .44 & .06 & .06 \\ \text { A5 } & .07 & .08 & .11 & .05 & .10 & .16 & .22 & .22 & .30 & .29 & .21 & .21 \\ \text { A8 } & .17 & .05 & .12 & .10 & .07 & .15 & .19 & .15 & .20 & .36 & .25 & .19 \\ \text { A9 } & .04 & .04 & .11 & .00 & .06 & .17 & .24 & .35 & .39 & .28 & .17 & .17\end{array}$


probabilities for each rat. Of the four summed measures, the two with the highest probabilities were found. For Rats A2, A3, A5, and A9 the tendency to perseverate was similar for the large and small reinforcers and the two measures together described the majority of the choice data. The summed perseveration probabilities were .57, .88, .59, and .67 for Rats A2, A3, A5, and A9, respectively. For these four rats, choice was not controlled by magnitude. Only Rat A8 showed a pattern of choice controlled by the position and magnitude of the most recent reinforcer. Probabilities were highest for perseverating on the lever that had recently produced a large reinforcer and for switching away from the lever that had recently produced a small reinforcer. These two probabilities summed to .61, describing a majority of choices for this rat. In other words, choices by Rat A8 followed a win-stay lose-shift pattern. After a large reinforcer, Rat A8 tended to choose the same lever again and, after a small reinforcer, the rat tended to choose the other lever. Because the position of the large reinforcer changed pseudorandomly, this pattern of responding was only slightly more effective in producing the large reinforcer than was exclusive preference, as indicated by the proportion of large choices under the 90-s ITI condition shown in Figure 7. Despite this control at the molecular level for one rat, choice responding was not under discriminative control of the cue-light stimuli for any rat. In fact, the data from four of five rats indicated that magnitude did not control choice behavior at all within the session.

For most rats, position bias alone predicted choice. For this reason, the procedure was modified to remove position bias as a hindrance to reliable preference for the large reinforcer by adopting a one-lever choice procedure.

Recall that the go/no-go procedure presented only choice trials. The cue light above the lever signaled the consequences of responding and not responding. By responding, the rat 
actively chose that (signaled) reinforcer. By not responding, the rat passively chose the other (unsignaled) reinforcer. The proportion of active choices and the proportion of large choices are shown in Figure 7. Although Rats A8 and A9 initially showed a small preference for the large reinforcer, by the end of the condition, choice proportions for the large reinforcer were close to the indifference point of .5 for all rats. The proportion of active choices was also similar across rats. Active choices were relatively frequent in the earliest sessions of the go/no-go procedure. With extended exposure to the procedure, the proportion of active choices dropped to extremely low levels. Thus, all rats learned not to press the lever during the choice period and this resulted in no preference for the large reinforcer. Casual observations of the rats during the final sessions of the go/no-go procedure revealed that the rats spent the majority of the session engaged in magazine-oriented behavior, with occasional interruptions by drinking behavior.

\section{Discussion}

The present experiment failed to establish a strong preference for the large reinforcer despite several procedural manipulations. The adjusting-delay procedure used in this study was adopted from the work of Mazur using pigeons (Mazur, 1987, 1988). It seems likely that the poorer visual acuity of albino rats may have produced these disappointing results. In addition to a failure of discrimination, the rats exhibited strong position biases that likely interfered with the development of discriminated performance based on magnitude. The uncanny ability of rats to respond on the basis of spatial stimuli is well-documented by a long list of maze experiments. This phylogenic predisposition may have been partly responsible for the failure of the present study. Indeed, researchers that have used adjusting-delay and similar procedures to study choice responding in rats have relied on spatial, rather than visual, discrimination (Richards, Mitchell, 
de Wit, \& Seiden, 1997; Ito \& Oyama, 1996; Logue, 1988; van Haaren et al., 1988). This arrangement was not possible in the present study because of the research question. To determine the delay at which differential reinforcer magnitudes have no differential reinforcing effect, it was necessary to ensure that the rats did not learn to respond simply on the large-magnitude lever at short delays and then continue choosing that lever at long delays, despite no sensitivity to reinforcer magnitude at long delays.

\section{Summary and Conclusions}

The present series of experiments was designed to determine whether operant behavior could be controlled by response-reinforcer relations that are temporally extended. In this vein, three such relations were studied. In Experiment 1, the relation between aggregated responses and aggregated reinforcers was examined. In Experiment 2, the relation between aggregated responses and a delayed reinforcer was examined. In Experiment 3, the relation between a choice response and a delayed reinforcer was examined. Together, these three experiments addressed the same general conceptual issue using different procedures.

Experiment 1 arranged bonus pellets at the end of 15-, 60- or 120-min sessions dependent on a shift in choice responding within the session. Although shifts in responding were observed, these shifts were not reliably related to the placement of the bonus contingency. The results were similar to those of Mellitz et al.(1983). Large individual differences tempered general conclusions regarding the efficacy of the response-reinforcer relation. For some rats, choice shifted in the appropriate direction in a majority of conditions, but not in all conditions, thus failing to provide conclusive evidence of control by the molar contingency.

Experiment 2 arranged much shorter delays to reinforcement than Experiment 1 and the 
delayed reinforcer was dependent on a short sequence of responses. To ensure that the delayed reinforcer was earned, a baseline schedule of immediate reinforcement was used to maintain moderate response rates. A discrete visual stimulus signaled the period during which responding would count toward the production of the delayed reinforcer. Elevated responding during the stimulus relative to responding prior to the onset of the stimulus gauged the reinforcing efficacy of the delayed reinforcer. Reliable elevations were observed for two rats at a delay of $40 \mathrm{~s}$ but not for the other two rats. Elevations observed at long delays may indicate sensitivity to the delayed reinforcer or merely the perseveration of elevated responding established at shorter delays. Overall, the results seem to support control of aggregated responses by an operant consequence delayed $40 \mathrm{~s}$ or less.

Experiment 3 attempted to determine the maximal delay at which differential reinforcer magnitude differentially reinforces choice behavior. Only one response was required to produce reinforcement and visual stimuli differentiated the responses that produced the small and large reinforcers. Even with short delays, discriminative control of choice by reinforcer magnitude was not established, despite several procedural variations designed to do so. The absence of discriminative control may have resulted from the use of visual discriminative stimuli as opposed to spatial ones. The poor visual acuity of albino rats and the phylogenic tendency to respond on the basis of position likely hindered the control of choice by the different response-reinforcer relations.

Experiment 1 was similar in some respects to the study by Bacotti (1976). Bacotti arranged a VI schedule in a 60-min session and manipulated the delay between the end of the session and presentation of the daily food ration. This large amount of food was certainly greater 
than the bonus pellets earned in Experiment 1. Furthermore, the food was delivered responseindependently at the end of every session. In Experiment 1, bonus pellets were only presented if choice behavior within the session shifted in the prescribed direction. This resulted in the delivery of bonus pellets at the end of about half of the sessions only. The probability of food presentation was therefore lower than in Bacotti's study. Infrequent contact with the bonus and the relatively small magnitude of the bonus may have reduced the likelihood of producing control by the post-session consequence in Experiment 1 .

Experiment 2 presented the bonus pellets more frequently and, overall, more pellets were earned by producing the bonus than by responding on the baseline schedule of immediate reinforcement. Furthermore, the bonus was delivered several times per session and the magnitude was six times larger than the immediate reinforcer. These procedural features likely enhanced sensitivity to the delayed consequence. The results were comparable in some ways to those from studies of response acquisition with delayed reinforcement (Critchfield \& Lattal, 1993; Lattal \& Gleeson, 1990; Lesage et al., 1996; Wilkenfield et al., 1992; Snycerski et al., 1999). Although in Experiment 2 responding was not established using delayed reinforcement, elevated responding during the initial-link was acquired by all rats under a 40-s delay to reinforcement (the first experimental condition). Shorter delays increased the degree of elevation. Only at short delays were elevations reliable for all rats. These results parallel those of the acquisition studies listed above in which reliably higher response rates (relative to control conditions with no reinforcement contingency) are observed for all rats only at short delays, usually $30 \mathrm{~s}$ and less.

Given the results of Timberlake et al. (1988), the time horizons observed in Experiments 1 and 2 seem quite short. This may be because Timberlake et al. failed to control the delay 
between the operant response and access to the rich (FR 1) schedule. Because rats had to cumulate a certain amount of time on the FR 1 side of the chamber, this response requirement virtually ensured that the rat was on the FR side when the FR schedule became available. Thus, obtained delays to reinforcement were likely quite short. This differs from Experiment 2 which controlled the temporal separation between effective responses and subsequent reinforcement. This seems to be a critical variable in studies of delayed reinforcement, because greater responsereinforcer contiguity is more likely to reinforce responding.

The data of Mellitz et al. (1983) prompted the present series of experiments. Their study suggested that aggregate response-reinforcer relations on the order of several minutes or more were effective in controlling the distribution of choice behavior within the session. Further, effective responses (those on the conjoint lever) were separated from the delayed reinforcer (session termination) by at least $2 \mathrm{~min}$. Only one of five rats preferred the conjoint lever across all four conditions. The authors asserted that this provided clear and convincing evidence of control by the conjoint contingency. However, in Experiment 1, which was analogous to their study in many respects, more reversals were conducted than by Mellitz et al., allowing a more stringent test of control by the molar response-reinforcer relation. For no rat did choice behavior shift in the direction of the bonus lever in all conditions, and across all rats, the number of appropriate shifts was close to that predicted by chance alone. Had Mellitz et al. conducted more reversals, their results might have been more similar to those in Experiment 1, and their conclusions regarding the reliability of control would have been more tempered.

Overall, the present series of experiments suggests that operant behavior in aggregate can be controlled by a delayed reinforcer, but finite limits to such control exist. The precise temporal 
window over which response-reinforcer relations are effective is likely to depend greatly on the exact procedures and parameters used. When delayed reinforcers are large in an absolute and relative sense, control by those reinforcers seems more likely. When frequent contact with the response-reinforcer relation is programmed, those relations seem more likely to control responding. Under conditions in which contact with the response-reinforcer relation is infrequent or the response-reinforcer relation is extended over a period of several minutes or more, control of operant behavior seems unlikely.

Answering questions regarding temporal limits of operant control will require diverse approaches and procedures. No single procedure will provide a definitive answer. The goal of future research in this area will be to continue devising experimental preparations that demonstrate behavioral control by temporally extended response-reinforcer relations. Particularly helpful in this regard would be those experiments that allow numerous parameters to be manipulated to determine under what conditions such control is and is not possible. This question must be addressed to provide a complete account of reinforcement operations and to settle conceptual issues in the areas of behavior analysis, behavioral ecology, ethology, and others. The applied implications of discovering how to improve control by temporally extended response-reinforcer relations are great. Numerous real-world problems could be reduced or eliminated by a fuller understanding of the conditions that produce molar control. 


\section{References}

Anger, D. (1963). The role of temporal discriminations in the reinforcement of Sidman avoidance behavior. Journal of the Experimental Analysis of Behavior, 6, 477-506.

Bacotti, A. V. (1976). Home cage feeding time controls responding under multiple schedules. Animal Learning \& Behavior, 4, 41-44.

Baum, W. M. (1973). The correlation-based law of effect. Journal of the Experimental Analysis of Behavior, 20, 137-153.

Belovsky, G. E. (1978). Diet optimization in a generalist herbivore: The moose. Theoretical Population Biology, 9, 129-136.

Critchfield, T. S., \& Lattal, K. A. (1993). Acquisition of a spatially-defined operant with delayed reinforcement. Journal of the Experimental Analysis of Behavior, 59, 373-387.

de Villiers, P. (1974).The law of effect and avoidance: A quantitative relationship between response rate and shock-frequency reduction. Journal of the Experimental Analysis of Behavior, 21, 223-235.

DeWaard, R. J., Galizio, M., \& Baron, A. (1979). Chained schedules of avoidance: Reinforcement within and by avoidance situations. Journal of the Experimental Analysis of Behavior, 32, 399-407.

Dinsmoor, J. A. (1977). Escape, avoidance, punishment: Where do we stand? Journal of the Experimental Analysis of Behavior, 28, 83-95.

Field, G. E., \& Boren, J. J. (1963). An adjusting avoidance procedure with multiple

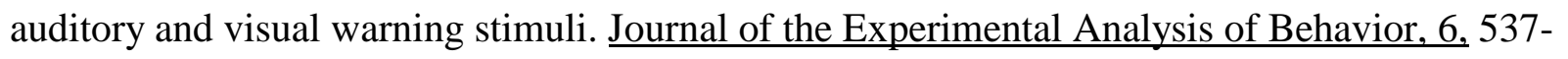
543. 
Fleshler, M., \& Hoffman, H. S. (1962). A progression for generating variable-interval schedules. Journal of the Experimental Analysis of Behavior, 5, 529-530.

Gormezano, I., Kehoe, E. J., \& Marshal, B. S. (1983). Twenty years of classical conditioning research with the rabbit. In J.M. Sprague \& A.N. Epstein (Eds.), Progress in Psychobiology and Physiological Psychology. Academic Press, New York, pp. 197-275.

Gormezano, I., \& Moore, J.W. (1969). Classical conditioning. In M.H. Marx (Ed.), Learning: Processes. MacMillan, New York, pp. 121-203.

Hackenberg, T. D., \& Axtell, S. A. M. (1993). Human's choices in situations of timebased diminishing returns. Journal of the Experimental Analysis of Behavior, 59, 445-470.

Herrnstein, R. J. (1969). Method and theory in the study of avoidance. Psychological Review, 76, 49-69.

Herrnstein, R. J. (1970). On the law of effect. Journal of the Experimental Analysis of Behavior, 13, 243-266.

Herrnstein, R. J. (1997). The matching law: Papers in psychology and economics. Edited by H. Rachlin \& D. I. Laibson. Cambridge, MA: Harvard University Press.

Herrnstein, R. J., \& Hineline, P. N. (1966). Negative reinforcement as shock-frequency reduction. Journal of the Experimental Analysis of Behavior, 9, 421-430.

Hineline, P. N. (1973). Negative reinforcement and avoidance. In W. K. Honig \& J. E. R. Staddon (Eds.), Handbook of operant behavior (pp. 364-414). Englewood Cliffs, NJ: PrenticeHall.

Hineline, P. N. (1981). The several roles of stimuli in negative reinforcement. In P. Harzem \& M. D. Zeiler (Eds.), Advances in analysis of behaviour: Vol. 2. Predictability, 
correlation, and contiguity (pp.203-246). Chichester, England: Wiley.

Hineline, P. N. (1984). Aversive control: A separate domain. Journal of the Experimental Analysis of Behavior, 42, 495-509.

Hineline, P. N., \& Sodetz, F. J. (1987). Appetitive and aversive schedule preferences: Schedule transitions as intervening events. In M. L. Commons, J. E. Mazur, J. A. Nevin, \& H. Rachlin (Eds.), Quantitative analyses of behavior: Vol. 5. The effect of delay and of intervening events on reinforcement value (pp. 144-157). Hillsdale, NJ: Erlbaum.

Hodos, W., \& Trumbule, G. H. (1967). Startegies of schedule preference in chimpanzees. Journal of the Experimental Analysis of Behavior, 10, 503-514.

Ito, M., \& Oyama, M. (1996). Relative sensitivity to reinforcer amount and delay in a self-control choice situation. Journal of the Experimental Analysis of Behavior, 66, 219-229.

Jacobs, E. A., \& Hackenberg, T. D. (1996). Human's choices in situations of time-based diminishing returns: Effects of fixed-interval duration and progressive-interval step size. Journal of the Experimental Analysis of Behavior, 65, 5-19.

Kamil, A. C. (1978). Systematic foraging by a nectar-feeding bird, the amakihi (Loxops virens). Journal of Comparative and Physiological Psychology, 92, 388-396.

Lattal, K. A., \& Gleeson, S. (1990). Response acquisition with delayed reinforcement. Journal of Experimental Psychology: Animal Behavior Processes, 16, 27-39.

Lattal, K. A., \& Metzger, B. (1994). Response acquisition by Siamese fighting fish (Beta $\underline{\text { splendens }) \text { with delayed visual reinforcement. Journal of the Experimental Analysis of Behavior, }}$ $\underline{61,35-44 .}$

LeSage, M. G., Byrne, T., \& Poling, A. (1996). Effects of $\underline{\mathrm{d}}$-amphetamine on response 
acquisition with immediate and delayed reinforcement. Journal of the Experimental Analysis of Behavior, 66, 349-367.

Logue, A. W. (1988). Research on self-control: An integrating framework. Behavioral and Brain Sciences, 11, 665-709.

Logue, A. W., \& de Villiers, P. (1978). Matching in concurrent variable-interval avoidance schedules. Journal of the Experimental Analysis of Behavior, 29, 61-66.

Lucas, G. A., Deich, J. D., \& Wasserman, E. A. (1981). Trace autoshaping: Acquisition, maintenance, and path dependence at long trace intervals. Journal of the Experimental Analysis of Behavior, 36, 61-74.

Mackintosh, N.J. (1974). The Psychology of Animal Learning. New York: Academic Press.

Mackintosh, N.J. (1983). Conditioning and Associative Learning. New York: Oxford University Press.

Mazur, J. E. (1987). An adjusting procedure for studying delayed reinforcement. In M. L. Commons, J. E. Mazur, J. A. Nevin, \& H. Rachlin (Eds.), Quantitative analyses of behavior: Vol. 5. The effect of delay and of intervening events on reinforcement value (pp. 55-73). Hillsdale, NJ: Erlbaum.

Mazur, J. E. (1988). Estimation of indifference points with an adjusting delay procedure. Journal of the Experimental Analysis of Behavior, 49, 37-47.

Mazur, J. E., \& Vaughan, W., Jr. (1987). Molar optimization versus delayed reinforcement as explanations of choice between fixed-ratio and progressive-ratio schedules. Journal of the Experimental Analysis of Behavior, 48, 251-261. 
McSweeney, F. K., Hatfield, J., \& Allen, T. M. (1991). Within-session responding as a function of post-session feedings. Behavioural Processes, 22(3), 177-186.

Mellitz, M., Hineline, P. N., Whitehouse, W. G., \& Laurence, M. T. (1983). Durationreduction of avoidance sessions as negative reinforcement. Journal of the Experimental Analysis of Behavior, 40, 57-67.

Myers, T. M., \& Perone, M. (1998). Assessing the limits of sensitivity to shock-frequency reduction as negative reinforcement. Unpublished manuscript, West Virginia University.

Neuman, P., Ahearn, W. H., \& Hineline, P. N. (2000). Pigeon's choices between fixedratio and linear or geometric escalating schedules. Journal of the Experimental Analysis of Behavior, 73, 93-102.

Newlin, R. J., \& Lolordo, V. M. (1976). A comparison of pecking generated by serial, delay, and trace autoshaping. Journal of the Experimental Analysis of Behavior, 25, 227-241.

Osborne, S.R., \& Shelby, M. (1975). Stimulus change as a factor in response maintenance with free food available. Journal of the Experimental Analysis of Behavior, 24, 17-21.

Richards, J. B., Mitchell, S. H., de Wit, H., \& Seiden, L. S. (1997). Determination of discount functions in rats with an adjusting-amount procedure. Journal of the Experimental Analysis of Behavior, 67, 353-366.

Rosen, R. (1967). Optimality principles in biology. Plenum, New York..

Schaal, D. W., \& Branch, M. N. (1990). Responding of pigeons under variable-interval schedules of signaled-delayed reinforcement: Effects of delay-signal duration. Journal of the Experimental Analysis of Behavior, 53, 103-121.

Schaal, D. W., \& Branch, M. N. (1988). Responding of pigeons under variable-interval 
schedules of unsignaled, briefly signaled, and completely signaled delays to reinforcement. Journal of the Experimental Analysis of Behavior, 50, 33-54.

Schoemaker, P. J. H. (1982). The expected utility model: Its variants, purposes, evidence and limitations. Journal of Economic Literature, 20, 529-563.

Shahan, T. A., \& Lattal, K. A. (1998). On the function of the changeover delay. Journal of the Experimental Analysis of Behavior, 69, 141-160.

Shull, R. L., Spear, D. J., \& Bryson, A. E. (1981). Delay or rate of food delivery as a determiner of response rate. Journal of the Experimental Analysis of Behavior, 35, 129-143.

Sidman, M. (1962). Reduction of shock frequency as reinforcement for avoidance behavior. Journal of the Experimental Analysis of Behavior, 5, 247-257.

Sizemore, O. J., \& Lattal, K. A. (1978). Unsignalled delay of reinforcement in variableinterval schedules. Journal of the Experimental Analysis of Behavior, 30, 169-175.

Snycerski, S., Laraway, S., Byrne, T., \& Poling, A. (1999). Acquisition of lever-press responding with delayed consequences in rats: Is a minute too long? Mexican Journal of Behavior Analysis, 25(3), 341-350.

Stubbs, D. A., \& Pliskoff, S. S. (1969). Concurrent responding with fixed relative rate of reinforcement. Journal of the Experimental Analysis of Behavior, 12, 887-895.

Timberlake, W., Gawley, D. J., \& Lucas, G. A. (1988). Time horizons in rats: The effect of operant control of access to future food. Journal of the Experimental Analysis of Behavior, 50, 405-417.

van Haaren, F., van Hest, A., \& van de Poll, N. E. (1988). Self-control in male and female rats. Journal of the Experimental Analysis of Behavior, 49, 201-211. 
Verhave, T. (1961). Some observations concerning prepotency and probability of postponing shock with a two-lever avoidance procedure. Journal of the Experimental Analysis of Behavior, 4, 187-192.

Wanchisen, B. A., Tatham, T. A., \& Hineline, P. N. (1988). Pigeon's choices in situations of diminishing returns: Fixed- versus progressive-ratio schedules. Journal of the Experimental Analysis of Behavior, 50, 375-394.

Wilkenfield, J., Nickel, M., Blakely, E., \& Poling, A. (1992). Acquisition of lever-press responding in rats with delayed reinforcement: A comparison of three procedures. Journal of the Experimental Analysis of Behavior, 58, 431-443.

Williams, B. A. (1976). The effects of unsignalled delayed reinforcement. Journal of the Experimental Analysis of Behavior, 26, 441-449. 